\title{
Zur mittel- und langfristigen Entwicklung der Erwerbstätigkeit in Deutschland
}

\author{
Axel Börsch-Supan · Christina Benita Wilke
}

Angenommen: 13. Mai 2008 / Online veröffentlicht: 10. März 2009

(C) Institut für Arbeitsmarkt- und Berufsforschung 2009

Zusammenfassung Dieses Papier legt Projektionen zur mittel- und langfristigen Entwicklung der Anzahl der Erwerbspersonen und Erwerbstätigen in Deutschland vor. Solche Projektionen hängen stark von zukünftigen Politikentscheidungen und den Verhaltensreaktionen auf sie ab. Diese sind kaum vorherzusagen. Wir gehen daher szenarienbasiert vor. Unser Hauptergebnis ist, dass in jedem Fall, auch dem günstigsten, sowohl die Erwerbspersonen- als auch die Erwerbstätigenanzahl künftig sinken wird.

Für unser vorsichtig optimistisches Szenario gehen wir von einer allmählichen Angleichung der deutschen Erwerbsquoten an die in Dänemark aus. In diesem Fall ergibt sich ein Rückgang in der Anzahl der Erwerbspersonen um etwa 2,8 Mio. auf etwa 39,4 Mio. im Jahr 2040. Ohne weitere Arbeitsmarktreformen würde die Anzahl der Erwerbspersonen bis 2040 um über 9 Mio. auf 32,6 Mio. zurückgehen. Für die Zahl der Erwerbstätigen ergibt sich ein moderaterer Rückgang von 2,5 Mio. auf 36,2 Mio. im Jahr $2040 \mathrm{im}$ Dänemark-Szenario und ein wesentlich stärkerer Rückgang von 11 Mio. auf 27,4 Mio. im Status-quo-Szenario.

Zugleich altert die Erwerbsbevölkerung. Das Durchschnittsalter wird bis zum Jahr 2040 von 40 auf knapp 42 Jahre ansteigen. Der Anteil der Erwerbspersonen im Alter 55+ wird von etwa $11 \%$ auf $20 \%$ im Jahr 2040 steigen.

\section{Medium- and long-term labor force trends in Germany}

Abstract This paper presents projections of the mediumand long-term labor force trends in Germany. Since such projections depend highly on future political decisions and corresponding behavioral changes, we apply a scenariobased approach. Our main findings are that the future size of the labor force and actual employment will decline in the future even in the most favorable scenario.

In our slightly optimistic scenario we assume a stepwise convergence of German labor force participation rates to the current Danish rates. In this case the size of the labor force will decline by around 2.8 million to approximately 39.4 million in 2040. Without any further labor market reforms, the size of the labor force will decline by more than 9 million to around 32.6 million in 2040. Regarding actual employment, the decline is more moderate in the Denmark scenario (by 2.5 million to 36.2 million in 2040) but much stronger in the Status quo scenario (by 11 million to 27.4 million in 2040).

At the same time, the labor force ages rapidly. We find an increase in the share of workers aged 55 and older from around $11 \%$ today to roughly $20 \%$ in 2040 .

\section{Einleitung}

Der demografische Wandel führt dazu, dass es immer mehr ältere und immer weniger jüngere Menschen gibt. Dies hat nicht nur sozialpolitische Konsequenzen, wie sie im Hinblick auf die Nachhaltigkeit der sozialen Sicherungssysteme in den vergangenen Jahren in der Öffentlichkeit verstärkt diskutiert wurden, sondern beeinflusst unser gesamtes Wirtschaftsgeschehen und wird in den kommenden Dekaden einen tiefgreifenden makroökonomischen

A. Börsch-Supan · C. B. Wilke ( $)$

Universität Mannheim, Mannheim Research Institute for the Economics of Aging (MEA),

L13, 17, 68131 Mannheim, Deutschland

E-Mail: wilke@mea.uni-mannheim.de 
Strukturwandel hervorrufen. Maßgeblich für das Ausmaß dieser sich abzeichnenden Entwicklung ist jedoch nicht nur das demografisch-zahlenmäßige Verhältnis der Älteren zu den Jüngeren in einer Gesellschaft. Für die ökonomischen Konsequenzen ist zudem die Beteiligung am Erwerbsleben wichtig. Dazu bedarf es Projektionen zur mittel- und langfristigen Entwicklung der Anzahl der Erwerbspersonen sowie der Erwerbstätigen in Deutschland. Dies ist das Thema dieses Beitrags.

Methodisch gehen unsere Projektionen zurück auf eine Erwerbstätigkeitsprognose für Deutschland von BörschSupan (2003). Neuere angebotsorientierte Arbeitsmarktprojektionen wurden für Deutschland 2005 vom Institut für Arbeitsmarkt- und Berufsforschung (IAB) (Fuchs u. Dörfler 2005a, b; Fuchs u. Söhnlein 2007), sowie von der Europäischen Kommission herausgegeben (Carone 2005). Eine eher nachfrageorientierte Arbeitsmarktprojektion erstellte die Kommission für die Nachhaltigkeit in der Finanzierung der Sozialen Sicherungssysteme (2003) (,Rürup-Kommission“). Allerdings basieren diese Studien auf älteren Bevölkerungsprognosen. Ziel dieses Beitrags ist es, von den aktuellsten Bevölkerungsvorausberechnungen des Statistischen Bundesamtes ausgehend die Spannweite plausibler Erwerbsprojektionen aufzuzeigen.

Fundament einer Projektion der Anzahl der Erwerbspersonen bzw. Erwerbstätigen ist zunächst die Demografie. Dieser Beitrag betont, dass die offiziellen Annahmen über die zukünftige Lebenserwartung zu pessimistisch sind, $d$. h. eine zu kurze Lebenszeit vorhersagen, wenn man sich an der vergangenen Entwicklung und den neuesten Ergebnissen der internationalen demografischen Forschung orientiert. Wir stellen daher zunächst verschiedene Szenarien der demografischen Entwicklung vor und wählen dasjenige, das uns am wahrscheinlichsten erscheint.

Die Entwicklung der Anzahl der Erwerbspersonen bzw. Erwerbstätigen wird nach der Demografie von den künftigen alters- und geschlechtsspezifischen Erwerbsquoten bestimmt. Diese hängen stark von zukünftigen Politikentscheidungen und die Verhaltensreaktionen auf sie ab. Sie sind kaum vorherzusagen. Wir gehen daher auch hier szenarienbasiert vor.

Wir wählen vier Szenarien, welche die Spannbreite möglicher künftiger Entwicklungen aufzeigen. Die Ober- und Untergrenzen sind leicht gesetzt. Nach oben hin begrenzt die durch die Demografie vorgegebene Bevölkerung im erwerbsfähigen Alter die Entwicklungsmöglichkeiten. In unserem optimistischen Szenario gehen wir davon aus, dass dieses Erwerbspersonenpotenzial bestmöglich ausgeschöpft wird. Die Untergrenze wird durch den Status quo markiert, d.h. die Annahme, dass sich die heutigen Erwerbsquoten auch in Zukunft nicht ändern werden. Ein Rückwärtstrend der Erwerbsquoten (und somit eine noch niedrigere Untergrenze) erscheint uns hingegen nicht plausibel.
Um ein realistisches Szenario innerhalb dieser Ober- und Untergrenzen zu erhalten und um abzuschätzen, wie weit das Erwerbspersonenpotenzial in realistischem Maße ausgeschöpft werden kann, ist ein Blick auf die europäischen Nachbarländer hilfreich, in denen ähnliche Arbeitsmarktreformen wie die deutschen stattgefunden haben. Wir wählen Dänemark als einen Referenzpunkt mit einer relativ hohen Erwerbsbeteiligung sowohl der Frauen als auch der Älteren aus. Unterstellt man einen Anstieg der deutschen Erwerbsquoten auf das dänische Niveau, so zeigt sich, dass ein Großteil des demografischen Drucks durch den Arbeitsmarkt abgefedert werden kann. Dies ist eine wichtige wirtschaftspolitische Botschaft.

Natürlich sind weitere Szenarien denkbar, vor allem ein Nachlassen der Reformtätigkeit, die jedoch nicht im vollständigen Stillstand unseres Status-quo-Szenarios endet. Wir modellieren dies mithilfe eines Szenarios, das wir „Slow-Go“ nennen. Dieses Szenario geht davon aus, dass die in der Agenda 2010 enthaltenen Reformen nur teilweise umgesetzt werden. Weitere Szenarien kann der Leser approximativ durch Interpolation unserer Ergebnisse bilden.

Der Beitrag gliedert sich in sechs Abschnitte. Abschnitt 2 beschreibt die zugrunde liegenden Bevölkerungsannahmen. In Abschn. 3 werden die zugrunde liegenden Annahmen sowie die Methodik der Arbeitsmarktprojektion erläutert. Abschnitt 4 präsentiert und interpretiert die Ergebnisse der verschiedenen Projektionen. Im Rahmen einer Sensitivitätsanalyse zeigt Abschn. 5 die Wirkung der einzelnen das Arbeitsangebot bestimmenden Parameter. Abschnitt 6 schließt mit Fazit und Ausblick.

\section{Annahmen zur künftigen Bevölkerungsentwicklung}

Projektionen der Erwerbspersonen- sowie Erwerbstätigenanzahl bauen auf Bevölkerungsprognosen auf. In diesem Abschnitt werden daher zunächst die Annahmen zur künftigen demografischen Entwicklung vorgestellt. Wir verwenden eigene Bevölkerungsprognosen, die auf der aktuellsten Prognose des Statistischen Bundesamtes aufsetzen. Basisjahr unserer Berechnungen ist das Jahr 2005; unser Prognosezeitraum erstreckt sich dann bis zum Jahr 2050.

11. Koordinierte Bevölkerungsvorausberechnung. Ausgangspunkt unserer Berechnungen ist die 11. koordinierte Bevölkerungsvorausberechnung des Statistischen Bundesamtes (2006). Als „mittleres Szenario“ werden dort zwei Varianten ausgewiesen: die Variante 1W1 als Unterund die Variante 1W2 als Obergrenze. Die erste Ziffer beschreibt dabei die zugrunde liegenden Annahmen hinsichtlich der Lebenserwartung, während die zweite Ziffer die Migrationsannahmen ( $\mathrm{W}=$ Wanderungen) beschreibt. Beide Varianten gehen von einer weiterhin stagnierenden 
Geburtenrate (1,4 Geburten pro Frau) aus. Beiden Varianten liegt die Annahme zugrunde, dass die Lebenserwartung für Männer auf 83,5 und für Frauen auf 88,0 Jahre im Jahr 2050 ansteigt. ${ }^{1}$ Der Unterschied zwischen den Varianten liegt in den Migrationsannahmen. Während die Variante 1W1 einen Wanderungssaldo von 100.000 (W1) unterstellt und damit den rückgängigen Trend der letzten Jahre aufgreift, basiert die Variante 1W2 auf dem für Deutschland zu beobachtenden langfristigen mittleren Wanderungssaldo von 200.000 (W2) Personen jährlich. Dazwischen gibt es kein weiteres Wanderungsszenario.

MEA-Bevölkerungsprojektionen. Ausgehend von dem Wunsch, ein „mittleres“ Wanderungsszenario zu verwenden und darüber hinaus eine aus unserer Sicht realistischere Entwicklung der Lebenserwartung $\mathrm{zu}$ modellieren, erstellen wir zwei eigene Bevölkerungsprojektionen. ${ }^{2}$ Als Referenzprojektion (mit „MEA 3W1,5“ bezeichnet) gehen wir von einer deutlich stärkeren Alterung bei mittlerer Immigration aus. $\mathrm{Zu}$ Vergleichszwecken berechnen wir zudem ein von der Lebenserwartung der 11. koordinierten Bevölkerungsvorausberechnung ausgehendes Szenario (,MEA 1W1,5“).

Im Einzelnen: Beide Szenarien nehmen die gleiche konstante Geburtenrate von 1,4 Geburten pro Frau wie die 11. koordinierte Bevölkerungsvorausberechnung an. ${ }^{3}$ Bei den Wanderungen wählen wir eine mittlere Variante zwischen den vom Statistischen Bundesamt ausgegebenen unteren und oberen Varianten und unterstellen einen langfristig mittleren Saldo von 150.000 Personen jährlich (daher die Abkürzung „W1,5“). Bei der Lebenserwartung schließlich gehen wir für unser Basisszenario (MEA 3W1,5) von einer Trendfortschreibung der vergangenen Jahrzehnte aus. Indem wir den nach dem Zweiten Weltkrieg beobachtbaren fast durchgehend linearen Trend getrennt für Männer und Frauen bis 2050 fortschreiben, ermitteln wir eine Lebenserwartung von 85,7 bzw. 91,7 Jahren für Männer bzw. Frauen im Jahr 2050. Unsere Annahmen zur Lebenserwartung liegen deutlich über der „Basisannahme“ bzw. leicht über dem „Hohen Anstieg“ des Statistischen Bundesamtes, die wir angesichts der vergangenen stetigen Unterschätzungen für unrealistisch halten. Schnabel et al. (2005) berechnen je

\footnotetext{
${ }^{1}$ In weiteren Varianten 2W1 bzw. 2W2 stellt das Statistische Bundesamt dieser „Basisannahme“ das Szenario „Hoher Anstieg“ (2) gegenüber, bei dem die Lebenserwartung für Männer auf 85,4 und für Frauen auf 89,8 Jahre im Jahr 2050 ansteigt.

${ }^{2} \mathrm{Zu}$ diesem Zweck wurde ein Bevölkerungssimulationsprogramm erstellt und so kalibriert, dass es bei gegebenen Annahmen die Ergebnisse der verschiedenen Varianten der Koordinierten Bevölkerungsvorausberechnung des Statistischen Bundesamtes (2006) reproduziert.

${ }^{3}$ Die Geburtenrate wirkt sich nur langfristig auf die Entwicklung der Erwerbspersonen aus. Erst nach 2050 machen sich Änderungen in der Geburtenrate deutlich bemerkbar.
}

nach Extrapolationsmethodik sogar eine Lebenserwartung von $92,6 \pm 3,8$ Jahre bzw. $94 \pm 2,8$ Jahre im Jahr 2050. In Anlehnung an die Notation des Statistischen Bundesamtes kennzeichnen wir unser Lebenserwartungsszenario mit der vorangestellten ersten Ziffer „3“.

Ein Überblick über die Annahmen der verschiedenen genannten Bevölkerungsprognosen findet sich in Tabelle 1. Als Vergleich ist dort neben den besprochenen Szenarien des Statistischen Bundesamtes und des MEA auch das Bevölkerungsszenario der Rürup-Kommission angegeben, weil dies nach wie vor vielfach verwendet wird, insbesondere weiterhin als Referenzpunkt in der Sozialpolitik dient.

Entwicklung der Gesamtbevölkerung. Abbildung 1 zeigt die Entwicklung der Gesamtbevölkerung bis 2050 gemäß den MEA-Bevölkerungsprognosen, den Varianten 1W1 und 2W2 des Statistischen Bundesamtes (StatBA) und dem Szenario der Rürup-Kommission. Interessanterweise heben sich die gegenläufigen Annahmen im MEABasisszenario (3W1,5) und der StatBA-Variante 1W2 ebenso wie im MEA-Alternativszenario $(1 \mathrm{~W} 1,5)$ und der StatBA-Variante 2W1 mit kleinen Abweichungen auf. Die Varianten 1W2 und 2W1 werden in Abb. 1 daher nicht extra dargestellt.

Ausgehend von einer Bevölkerung von 82,4 Mio. Einwohnern im Jahr 2005 führt das MEA-Basisszenario (MEA 3W1,5) zu einem Rückgang um etwa 8 Mio. auf 74,4 Mio. Einwohner 2050. Dies entspricht den Werten des Rürup-Szenarios zum Endpunkt unserer Projektionen im Jahr 2050. Das Rürup-Szenario unterstellt im Gegensatz zu den neueren Prognosen jedoch eine Zunahme der Bevölkerung bis 2020. Geringer ist der Bevölkerungsrückgang bis 2050 in der StatBA-Variante 2W2, die eine deutlich höhere jährliche Nettomigration zugrunde legt. Im MEAAlternativszenario (MEA 1W1,5) ergibt sich ein wesentlich stärkerer Bevölkerungsrückgang um knapp 11,3 Mio. auf 71,1 Mio. Einwohner 2050. Die Variante 1W1 des

Tabelle 1 Bevölkerungsprognosen im Überblick

\begin{tabular}{lllll}
\hline & $\begin{array}{l}\text { Lebenserwartung } \\
\text { Neugeborener }\end{array}$ & $\begin{array}{l}\text { Wanderungs- } \\
\text { saldo (in Tsd.) }\end{array}$ & $\begin{array}{l}\text { Geburtenrate } \\
\text { (Geburten/Frau) }\end{array}$ \\
\hline Rürup-Komm. & M: 81,3 & F: 86,6 & 200 & \\
StatBA 1W1 & M: 83,5 & F: 88,0 & 100 & \\
MEA 1W1,5 & & & 150 & \\
StatBA 1W2 & & & 200 & 1,4 \\
StatBA 2W1 & M: 85,4 & F: 89,8 & 100 & \\
StatBA 2W2 & & & 200 & \\
MEA 3W1,5 & M: 85,7 & F: 91,7 & 150 & \\
\hline
\end{tabular}

Quelle: Eigene Darstellung basierend auf der 11. koordinierten Bevölkerungsvorausberechnung des Statistischen Bundesamtes (2006) und dem demografischen Szenario der Kommission für die Nachhaltigkeit in der Finanzierung der Sozialen Sicherungssysteme (2003). 
Abb. 1 Bevölkerungsprojektionen im Vergleich. Quelle: Eigene Berechnungen auf Basis von Daten des Statistischen Bundesamtes (www.destatis.de), Daten der 11. koordinierten Bevölkerungsvorausberechnung des Statistischen Bundesamtes (2006) sowie dem demografischen Szenario der Kommission für die Nachhaltigkeit in der Finanzierung der Sozialen Sicherungssysteme (2003)

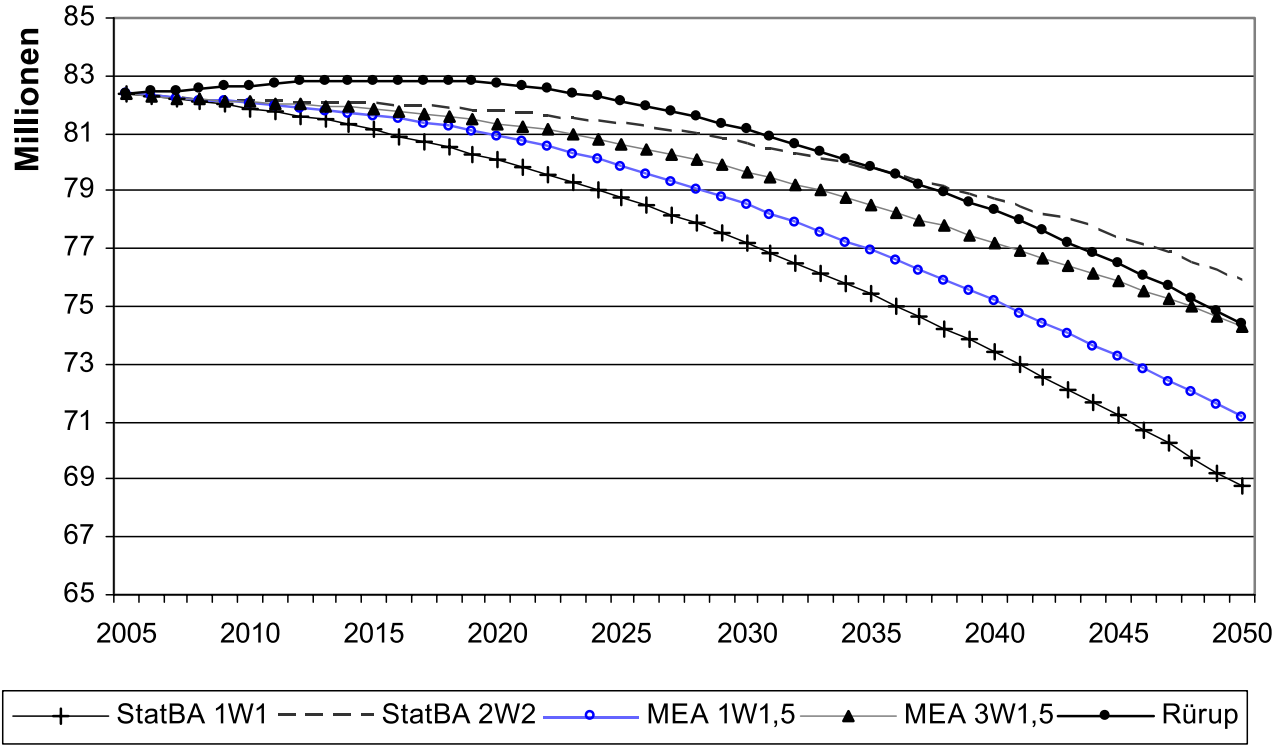

Statistischen Bundesamtes liegt mit 68,8 Mio. im Jahr 2050 nochmals um gute 2,3 Mio. darunter.

Veränderungen in der Altersstruktur. Das Ausmaß des allgemeinen Bevölkerungsrückgangs ist jedoch wenig relevant für die Entwicklung des Pro-Kopf-Einkommens oder der Nachhaltigkeit der Sozialversicherungssysteme. Zentral hierfür ist vielmehr die Entwicklung der Bevölkerung im erwerbsfähigen Alter relativ zur Entwicklung der Gesamtbevölkerung. Abbildung 2 zeigt daher die Entwicklung des Altersquotienten für die oben vorgestellten Prognosevarianten. Er ist definiert als das Verhältnis der Anzahl der über 64-Jährigen zur Anzahl der 15- bis 64-Jährigen.
Die Änderung der Altersstruktur ist einschneidender als der Bevölkerungsrückgang, da der prozentuale Rückgang der Personen im erwerbsfähigen Alter wesentlich größer ist als derjenige der Gesamtbevölkerung. Im MEABasisszenario (3W1,5) steigt daher der Altersquotient von 0,29 im Jahr 2005 auf 0,65 im Jahr 2050, was mehr als einer Verdoppelung entspricht, d.h. einer Person im Alter $65+$ werden in diesem von uns als wahrscheinlich eingeschätzten Szenario im Jahr 2050 noch etwa 1,5 Personen im erwerbsfähigen Alter zwischen 15 und 64 Jahren gegenüber stehen. Der Anstieg des Altersquotienten verläuft im MEA-Alternativszenario (1W1,5) etwas moderater auf 0,58 im Jahr 2050. Bei den StatBA-Varianten 1W1
Abb. 2 Entwicklung des Altersquotienten. Quelle: Eigene Berechnungen auf Basis von Daten des Statistischen Bundesamtes (www.destatis.de), Daten der 11. koordinierten Bevölkerungsvorausberechnung des Statistischen Bundesamtes (2006) sowie dem demografischen Szenario der Kommission für die Nachhaltigkeit in der Finanzierung der Sozialen Sicherungssysteme (2003)

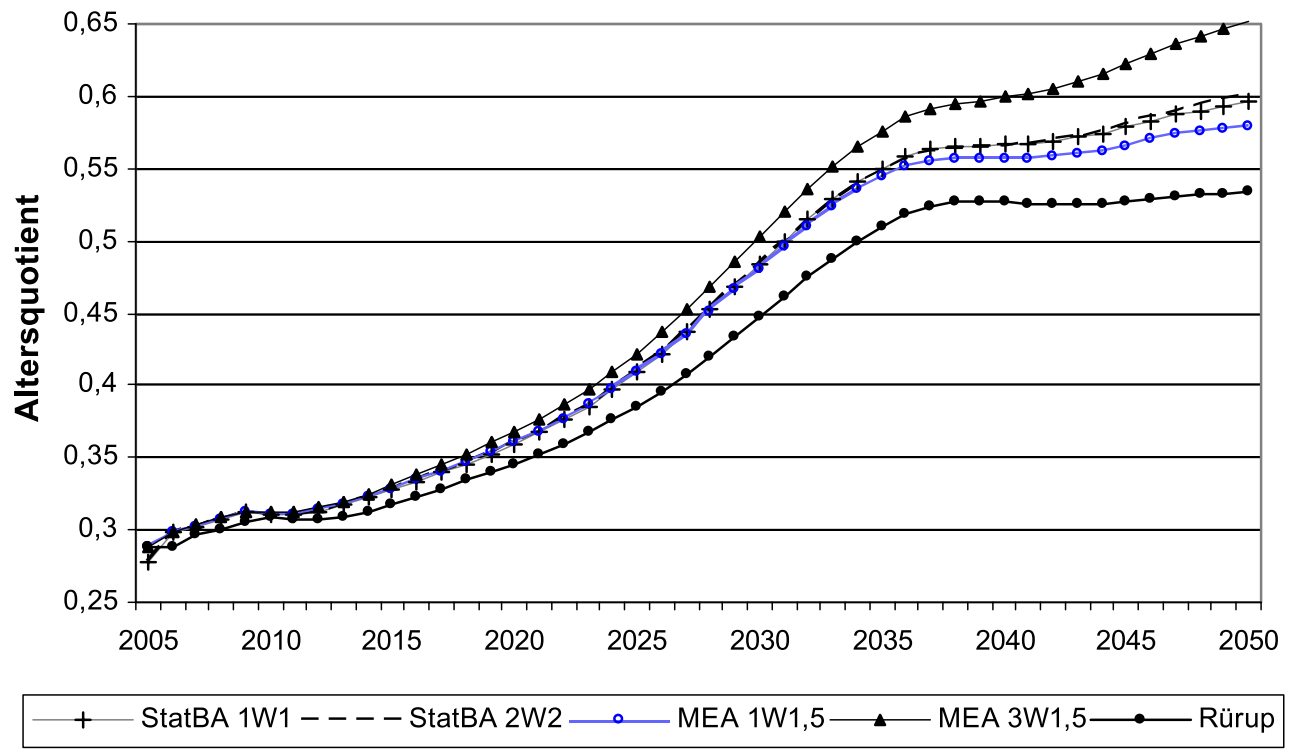


und 2W2 ergibt sich bis 2030 ein nahezu identischer Anstieg des Altersquotienten wie im MEA-Alternativszenario. Der Altersquotient nimmt danach aber nochmals zu, sodass der Altersquotient 2050 etwas über dem MEAAlternativszenario liegt. Die optimistischste Entwicklung zeigt das Rürup-Szenario, bei dem der Altersquotient bis 2050 ,nur“ auf 0,53 ansteigt.

Im Gegensatz zur Entwicklung der Gesamtbevölkerungszahl, entspricht die Entwicklung des Altersquotienten im MEA-Basisszenario nun der Variante 2W1, während die Altersstruktur im MEA-Alternativszenario relativ mittig bezüglich der beiden Varianten 1W2 und 2W2 der 11. koordinierten Bevölkerungsvorausberechnung liegt. Dies zeigt, dass sich die MEA-Bevölkerungsprojektionen im Hinblick auf die Altersstruktur, die für die Abschätzung des Erwerbspotenzials fundamental ist, deutlich von den Varianten der 11. koordinierten Bevölkerungsvorausberechnung unterscheiden.

\section{Modellierung von Arbeitsangebot und Arbeitsnachfrage}

Arbeitsangebot. Mit dem demografischen Wandel sinkt die Anzahl der Personen im erwerbsfähigen Alter, das sogenannte Erwerbspersonenpotenzial. Inwieweit sich diese Entwicklung auf die tatsächliche Anzahl der Erwerbspersonen (Erwerbstätige + Arbeitslose) und damit das Arbeitsangebot auswirkt, hängt davon ab, wie gut das Erwerbspersonenpotenzial ausgeschöpft werden kann, d. h. wie viele Personen der "stillen Reserve“ (der Differenz zwischen Erwerbspersonenpotenzial und Erwerbspersonen) tatsächlich Arbeit anbieten. Zur stillen Reserve zählen auch Ausländer, die in den deutschen Arbeitsmarkt eintreten wollen. Sie sind implizit in unseren Wanderungsannahmen enthalten.

Arbeitsnachfrage. Nicht alle Erwerbspersonen finden jedoch Arbeit. Für die langfristige Entwicklung der Arbeitsnachfrage spielen die Kapital- und Arbeitskosten (Löhne, Lohnnebenkosten und Steuern, vor allem im internationalen Vergleich) und strukturelle Eigenschaften des Arbeitsmarktes (die sich u. a. in Einstellungs- und Kündigungskosten niederschlagen) die entscheidende Rolle, für die kurzfristige Nachfrage auch die Konjunktur.

Modellierungsansatz. In diesem Papier wird bewusst von einer strukturellen Modellierung der Arbeitsnachfrage abgesehen. ${ }^{4}$ Zum einen wollen wir uns auf mittel- und langfristige Trends konzentrieren und ignorieren daher Schwankungen über den konjunkturellen Verlauf. Unsere

${ }^{4}$ Für eine aktuelle Projektion des Arbeitskräftebedarfs bis 2020 siehe Schnur u. Zika (2005).
Projektionen stellen damit langfristig gleitende Mittelwerte über die Konjunkturzyklen dar. Zum zweiten modellieren wir die konjunkturunabhängige, langfristige Arbeitslosigkeit als Szenarien, die sich aufgrund struktureller Parameter, insbesondere der Arbeitsmarktpolitik, ergeben. Die Anzahl der Erwerbstätigen ergibt sich dann als Anzahl der Erwerbspersonen minus Anzahl der Arbeitslosen. Wir treffen implizit somit folgende Annahmen:

1. Veränderungen der Arbeitsnachfrage wirken ausschließlich über die Arbeitslosenquote (ALQ). Wir geben diese szenarisch vor.

2. Änderungen im Arbeitsangebotsverhalten beeinflussen die Arbeitslosenquote nicht, wohl aber die Arbeitslosenzahl: Jedes zusätzliche Arbeitsangebot wird vom Arbeitsmarkt im Umfang von (1-ALQ) absorbiert.

\subsection{Modellparameter}

Den Projektionen der künftigen Anzahl der Erwerbstätigen, Erwerbslosen und Rentner legen wir in einem ersten Schritt alters- und geschlechtsspezifische Erwerbsquoten ${ }^{5}$ zugrunde, deren Entwicklung im Zeitablauf durch eine Reihe verhaltens- und arbeitsmarktpolitischer Parameter bestimmt wird. In einem zweiten Schritt multiplizieren wir diese Erwerbsquoten mit den alters- und geschlechtsspezifischen Belegungszahlen der Bevölkerungsprognose. ${ }^{6}$ Die Vorgehensweise, zunächst die „tiefen“ strukturellen Parameter zu spezifizieren, und diese dann szenarisch mit bestimmten Werten zu füllen (siehe den folgenden Abschn. 3.2), sichert die interne Konsistenz der von uns gemachten Annahmen. Wir berücksichtigen in unserem Modell Veränderungen in drei wesentlichen das Arbeitsangebot bestimmenden Parametern (Berufseintrittsalter, Frauenerwerbsquote und Renteneintrittsalter), sowie Veränderungen in der Arbeitslosenrate, die implizit die Arbeitsnachfrage abbildet. Eine Änderung einer oder mehrerer dieser Parameter hat einen direkten Einfluss auf die Höhe der alters- und geschlechtsspezifischen Erwerbsquoten und wirkt somit unmittelbar auf das Arbeitsangebot bzw. die Arbeitsnachfrage.

\footnotetext{
${ }^{5}$ Anteil der Erwerbspersonen an der Bevölkerung. Unsere Erwerbsquoten basieren auf Daten des Mikrozensus des Statistischen Bundesamtes (www.destatis.de). Aufgrund der Untererfassung marginaler Erwerbstätigkeiten im Mikrozensus wird das Niveau der Gesamterwerbstätigkeit dort allerdings unterschätzt. Wir kalibrieren unsere Erwerbsquoten daher so, dass sie multipliziert mit der Bevölkerung für das Basisjahr die Anzahl der Erwerbspersonen gemäß der aggregierten Erwerbsquote der OECD Labour Statistics (http://www.oecd.org/topicstatsportal/0,3398, en_2825_495670_1_1_1_1_1,00.html\#499783) korrekt abbilden.

${ }^{6}$ Wir verzichten hier bewusst auf eine Differenzierung nach Ost und West, nicht zuletzt, weil eine substanzielle Vorausschätzung der entsprechenden Parameter schwierig erscheint. Zu Unterschieden im Erwerbsverhalten zwischen Ost und West siehe bspw. Fuchs u. Söhnlein (2007).
} 
Herabsetzung des Berufseintrittsalters. Der erste Parameter ist die Anzahl der Jahre, um die sich das mittlere Berufseintrittsalter verjüngt. Modelltechnisch werden dazu die Erwerbsquoten während der Ausbildungsphase bis zum Alter 27 um diese Anzahl an Jahren nach vorne verschoben. Zwischen dem Alter 27 und dem neuen Alter, das nun die Ausbildungsphase beendet, werden die Erwerbsquoten denen im Alter 27 angeglichen. Das früheste Berufseintrittsalter, für das im Mikrozensus eine Erwerbstätigkeit registriert wird, liegt bei 15 Jahren. Da ein früherer Eintritt in das Berufsleben unrealistisch ist, wird dieses Eintrittsalter beibehalten und es werden ihm lediglich die höheren Erwerbsquoten zugewiesen. Die zeitliche Anpassung der alters- und geschlechtsspezifischen Erwerbsquoten vom Basisjahr bis zum Zieljahr der Prognose erfolgt linear, indem die Differenz zwischen dem Basisjahr und dem Zieljahr gleichmäßig auf alle Prognosejahre aufgeteilt wird.

Erhöhung der Frauenerwerbsquote. Der zweite Parameter ist die altersspezifische Angleichung der Frauenerwerbsquoten an die der Männer. Wir modellieren diese Anpassung, indem wir die Differenz zwischen den Erwerbsquoten der Männer und Frauen um einen bestimmten Faktor verringern. Wieder erfolgt die zeitliche Anpassung zwischen den Erwerbsquoten im Basis- und im Zieljahr linear.

Erhöhung des Renteneintrittsalters. Der dritte Parameter ist das mittlere Renteneintrittsalter, das wir für die Zwecke dieses Beitrags mit dem mittleren Alter des Berufsausstiegs gleichsetzen. Wir modellieren eine Erhöhung dieses Alters, indem wir die Erwerbsquoten ab dem Alter 47, zu dem die altersspezifischen Erwerbsquoten ihren Höhepunkt erreicht haben, um die dem jeweiligen Szenario entsprechende Anzahl an Jahren nach hinten verschieben. Zwischen dem Alter 47 und dem neuen Alter, ab dem nun nach und nach der Übergang in die Rente stattfindet, werden die Erwerbsquoten denen im Alter 47 angeglichen (verschiebt sich das mittlere Renteneintrittsalter um ein Jahr nach hinten, wäre dies bspw. das Alter 48). Das mittlere Renteneintrittsalter liegt derzeit bei etwa 62 Jahren, sodass eine Verschiebung um zwei Jahre ein mittleres Renteneintrittsalter von etwa 64 Jahren bedeutet. Die zeitliche Anpassung der alters- und geschlechtsspezifischen Erwerbsquoten vom Basisjahr bis zum Zieljahr der Prognose erfolgt linear.

Reduktion der Erwerbslosenquote. Der vierte in den folgenden Szenarien zu spezifizierende Parameter ist schließlich die Erwerbslosenquote. ${ }^{7}$ Modelltechnisch nehmen wir

\footnotetext{
${ }^{7}$ Anteil der Erwerbslosen an den Erwerbspersonen (Erwerbstätige plus Erwerbslose). Wir verwenden die Definition der Erwerbslosenquote der Bun-
}

an, dass ein den Szenarien entsprechender Anteil der in der Vorperiode arbeitslosen Personen in der Folgeperiode den Sprung in die Erwerbstätigkeit schafft. Dabei wird von einer konstanten Altersverteilung der Erwerbslosen gemäß Abb. 3 sowie einer über alle Altersjahre gleichmäßigen Reduktion der Arbeitslosigkeit ausgegangen. Die zeitliche Anpassung der alters- und geschlechtsspezifischen Erwerbslosenquoten vom Basisjahr bis zum Zieljahr erfolgt linear.

\subsection{Szenarien}

Mithilfe der im vorigen Abschnitt erläuterten Parameter lässt sich nahezu jede künftige Arbeitsmarktentwicklung modellieren. Eine Abschätzung ihrer realistischen Spannbreiten hängt stark von der Einschätzung ab, welche zukünftigen arbeitsmarktrelevanten Politikentscheidungen gefällt und welche Verhaltensreaktionen auf sie stattfinden werden. Um eine solche Einschätzung transparent zu strukturieren, gehen wir, wie in der Einleitung betont wurde, szenarienbasiert vor. Im Folgenden stellen wir vier Szenarien vor (,Status quo“, „Best Case“, „Dänemark“, „Slow-Go“), welche die im vorangegangenen Abschnitt aufgeführten tiefen Parameter mit Zahlen füllen. Wie bei allen Szenarien mag man sich darüber streiten, wie wahrscheinlich sie eintreffen. Unsere vier Szenarien orientieren sich daher an plausiblen Referenzpunkten, welche die Spannbreite möglicher Entwicklungen aufzeigen. Durch Interpolation kann der geneigte Leser sich ausmalen, wo alternative Szenarien liegen.

Szenario „Status quo“. Ein erster wichtiger Referenzpunkt für eine strukturierte Analyse ist der Status quo. In diesem Szenario wird davon ausgegangen, dass die heutigen alters- und geschlechtsspezifischen Erwerbsquoten auch in Zukunft bestehen bleiben. Dieses Szenario unterstellt folglich, dass sich das mittlere Berufseintrittsalter nicht nach vorne verschiebt und sich die Ausbildungsdauer nicht verkürzt, dass sich die Frauenerwerbsquote nicht an die der Männer angleicht und dass die aktuellen Reformen im Bereich der Rentenversicherung keine weitere Erhöhung des mittleren Rentenzugangsalters bewirken und dass trotz des demografischen Wandels und des daraufhin zu erwartenden Arbeitskräftemangels es zu keiner Verringerung der Arbeitslosigkeit kommt. Wir halten diese Entwicklung für völlig unwahrscheinlich; das Szenario hat jedoch eine wichtige Aufgabe als leicht zu analysierende Untergrenze.

Szenario „Best Case“. Das Best-Case-Szenario dient dementsprechend als plausibles Modell einer Obergrenze. Es

desagentur für Arbeit, die sich auf alle zivilen Erwerbspersonen bezieht. Für das Basisjahr 2005 ergibt sich hiernach eine Erwerbslosenquote von etwa $9 \%$. 
Abb. 3 Alters- und geschlechtsspezifische Arbeitslosenquoten. Quelle: Eigene Berechnungen auf Basis von Daten des Statistischen Bundesamtes (www.destatis.de)

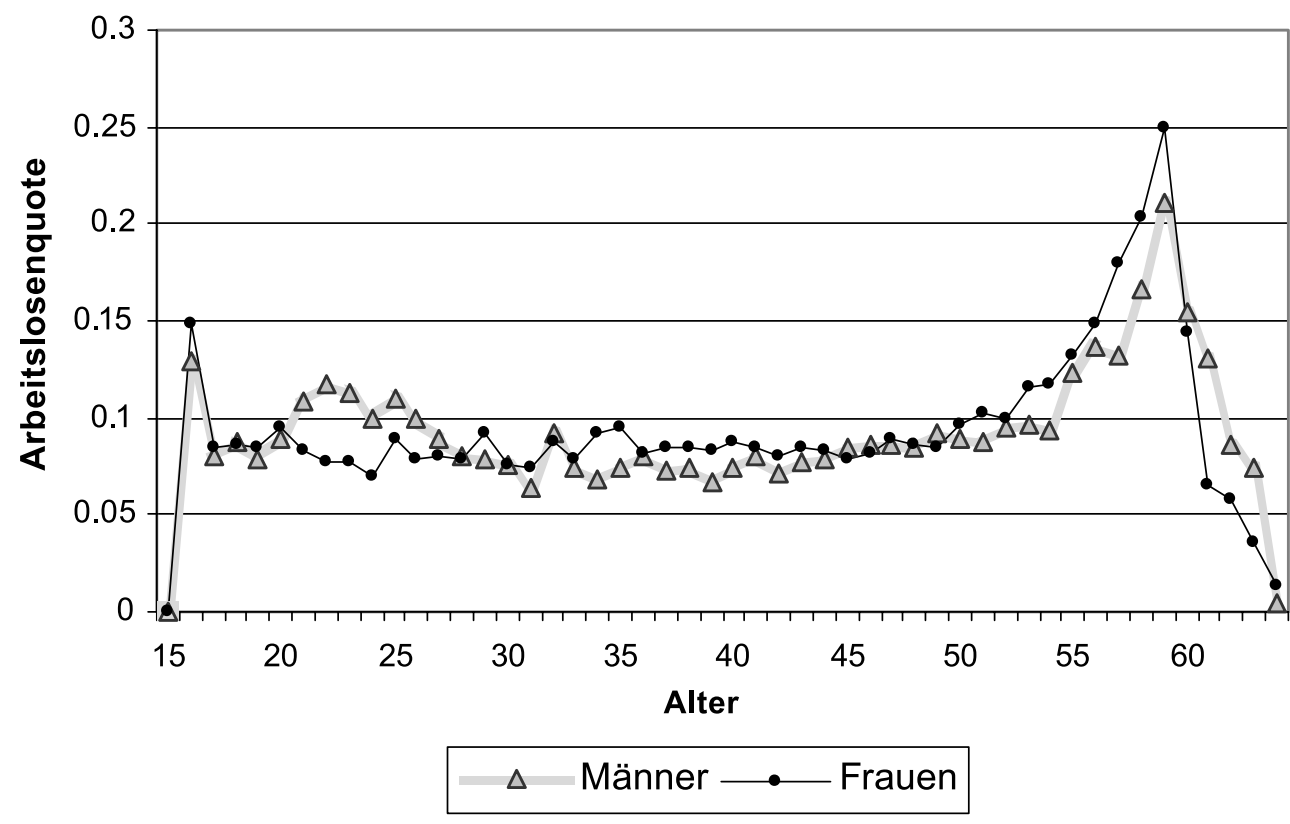

geht davon aus, dass sich bis 2040 das mittlere Berufseintrittsalter um zwei Jahre nach vorne verschiebt, dass sich die Frauenerwerbsquote nahezu vollständig (um 90\%) an die der Männer angleicht, die Erwerbsquote der Männer sich im Gegenzug auf 95\% reduziert und dass sich das mittlere Renteneintrittsalter um drei Jahre nach hinten verschiebt und dass sich die Arbeitslosigkeit bis 2040 auf das langfristig natürliche Niveau von etwa $4 \%$ reduziert. $^{8}$ Dieses Szenario orientiert sich an den Zielgrößen des US-amerikanischen Council of Economic Advisors. Wir halten eine so hohe Erwerbsbeteiligung in Deutschland auch langfristig für unrealistisch, da das Freizeitverhalten der Deutschen auch historisch gesehen stark von dem der US-Amerikaner abweicht. Das Szenario soll vielmehr verdeutlichen, welche Entwicklung auf dem Arbeitsmarkt zu erzielen wäre, wenn man in Deutschland die höchsten Beschäftigungsquoten eines OECD-Landes erreichen würde.

Szenario „Dänemark“‘. Ein realistischer Referenzpunkt für auch in Deutschland mögliche Arbeitsmarktveränderungen scheint uns Dänemark zu sein. Dieses Szenario orientiert sich daher an den heutigen alters- und geschlechtsspezifischen Erwerbsquoten in Dänemark. Die Unterschiede zwischen den heutigen deutschen (D) und dänischen (DK) Erwerbsquoten zeigt Abb. 4. Sie zeigt, dass die dänischen Erwerbsquoten der Frauen in allen Altersjahren deutlich oberhalb denen der deutschen Frauen liegen. Bei den Männern sind die Erwerbsquoten beider Länder in den

\footnotetext{
${ }^{8}$ Nach der Theorie von Milton Friedman (1968) schwankt die Arbeitslosenquote einer Volkswirtschaft langfristig um eine natürliche, positive Arbeitslosenquote. In der Literatur wird diese in der Regel bei $4 \%$ angenommen.
}

jungen Jahren etwa gleich. In den späteren Altersjahren bis zum Renteneintritt liegen die Erwerbsquoten der dänischen Männer hingegen deutlich oberhalb der Erwerbsquoten der deutschen Männer.

Das Dänemark-Szenario modelliert eine graduelle Angleichung der deutschen an die dänischen Erwerbstätigenquoten. Diese Angleichung vollzieht sich sehr langsam bis zum Jahr 2040. Die daraus resultierende Arbeitsangebotsentwicklung entspricht einer Herabsetzung des mittleren Berufseintrittalters um ein Jahr, einer Angleichung der Frauenerwerbstätigkeit an die der Männer um $90 \%$ bei entsprechender Reduktion der Männererwerbstätigkeit auf 95\%, einer Heraufsetzung des mittleren Renteneintrittsalters um zwei Jahre sowie einer Reduktion der Erwerbslosenquote in Deutschland auf das heutige dänische Niveau von $4,8 \%$.

Dänemark wurde vor allem deswegen als Referenzpunkt gewählt, weil die dortigen Strukturreformen eine wichtige Rolle bei der Entwicklung der sogenannten Agenda 2010 gespielt haben. ${ }^{9}$ Tatsächlich würden die in der Agenda 2010 zusammengefassten Reformschritte zu einer Beschäftigungssituation führen, die der heutigen dänischen in etwa entspricht. Dieses Szenario bildet daher eine realistische

\footnotetext{
${ }^{9} \mathrm{Zu}$ den beschlossenen Reformen der Agenda 2010 im Bereich des Arbeitsmarktes zählen insbesondere (1) die Kürzung des Leistungsanspruchs auf Arbeitslosengeld auf generell 12 Monate und 18 Monate für Menschen ab 55 Jahren, (2) die Abschaffung der Arbeitslosenhilfe zugunsten eines Arbeitslosengeldes II auf Sozialhilfeniveau, (3) eine verschärfte Zumutbarkeit für Arbeitsangebote sowie (4) der Zugang von vormals Sozialhilfeempfängern zu den Fördermaßnahmen der Bundesagentur für $\mathrm{Ar}$ beit. Für weitere Informationen zur Agenda 2010 (2003) siehe http:// archiv.bundesregierung.de/artikel/81/557981/attachment/557980_0.pdf.
} 
Abb. 4 Deutsche und dänische Erwerbsquoten im Vergleich. Quelle: Eigene Berechnungen auf Basis von Daten aus dem Mikrozensus des Statistischen Bundesamtes (www.destatis.de) und Statistics Denmark (www.statbank.dk)

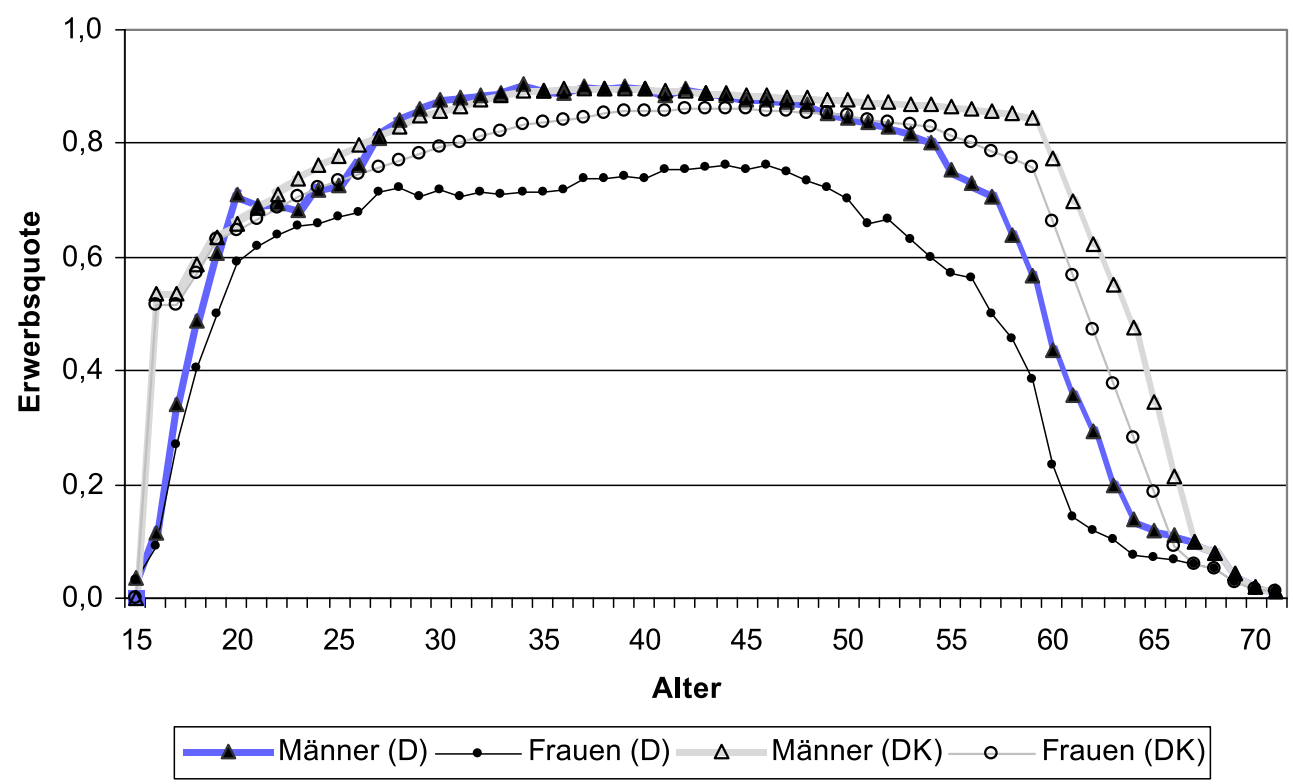

Entwicklung unter der Annahme ab, dass die Reformen der Agenda 2010 in einem sehr moderaten Tempo, aber letztlich konsequent, fortgeführt werden. ${ }^{10}$

Szenario ,Slow-Go“. Schließlich modellieren wir noch ein viertes Szenario, das von einer pessimistischeren Einschätzung in Deutschland möglicher Strukturreformen ausgeht. Um auch hier eine einfache und transparente Spezifikation vorzunehmen, die sich an bekannten Referenzpunkten orientiert, wählen wir eine Halbierung aller Veränderungen, die dem Dänemark-Szenario zugrunde lagen. Wir unterstellen also bis zum Jahr 2040 eine Erhöhung des mittleren Renteneintrittsalters um nur noch ein Jahr, einer Angleichung der Frauenerwerbsquote um lediglich $75 \%$ an die der Männer, während die Erwerbsquoten der Männer unverändert bleiben, eine Verschiebung des mittleren Berufseintrittsalters um ein Jahr nach vorne und einen langfristigen Rückgang der konjunkturbereinigten Arbeitslosenquote von 9 auf $7 \%$.

\section{Ergebnisse der Arbeitsmarktprojektionen}

Unsere Simulationen gehen vom Basisjahr 2005 aus, für das die letzten historischen Bevölkerungsdaten vorliegen. Sie beinhalten bis zum Jahr 2008 jedoch noch keinerlei Veränderungen in den Erwerbsquoten, da die für die Szenarien „Dänemark“ und „Best Case“ unterstellten und

\footnotetext{
${ }^{10}$ Diesem Szenario liegt die eher moderate Annahme zugrunde, dass Deutschland in den kommenden 30 Jahren diejenigen Arbeitsmarktreformen durchführen kann, die Dänemark in den letzten 10 Jahren durchgeführt hat.
}

über die Agenda 2010 hinausgehenden Arbeitsmarktreformen prinzipiell erst ab 2008 Wirkung zeigen können. Die Projektionen gehen bis zum Jahr 2040. Danach halten wir die Erwerbsquoten konstant. Veränderungen im Arbeitsangebot begründen sich dann nur noch durch die zugrunde liegende Demografie. Um auch diese Entwicklung aufzuzeigen, stellen wir unsere Ergebnisse der Projektionen in den Abbildungen bis zum Jahr 2050 dar. Die zugrunde liegende Bevölkerungsprojektion ist unser Basisszenario MEA 3W1,5.

\subsection{Erwerbspersonen und Erwerbstätige}

Die Anzahl der Erwerbspersonen geht in allen vier Szenarien zurück (Abb. 5), wenn auch das Ausmaß des Rückgangs sehr unterschiedlich ausfällt. Während im Status quo ein Rückgang von 9,6 Mio. (etwa 22,7\%) auf etwa 32,6 Mio. Erwerbspersonen $2040 \mathrm{zu}$ verzeichnen ist, sinkt die Anzahl der Erwerbspersonen bis 2040 im Szenario Slow-Go um 5,8 Mio. auf 36,4 Mio. (etwa 13,7\%), im Dänemark-Szenario um 3,9 Mio. (etwa 9,2\%) auf knapp 38,3 Mio. und im Best-Case-Szenario um 2,8 Mio. auf nur 39,4 Mio. (etwa 6,6\%). Bis 2040 wird es im DänemarkSzenario somit fast 4 Mio. weniger Erwerbspersonen als im Jahr 2005 geben. Das Arbeitsangebot wird also, absolut gesehen, um etwa die heutige Zahl der Arbeitslosen schrumpfen.

Ab 2040 nimmt der Rückgang der Erwerbspersonenanzahl zu und entspricht dem Tempo im Status quo. In den Szenarien „Best Case“ und „Dänemark“ entsteht ein Knick, da die Erwerbsquoten nach 2040 annahmegemäß ihren langfristigen Wert erreicht haben, also nach 2040 nicht weiter steigen, die Bevölkerung aber weiterhin sinkt und altert. 
Abb. 5 Entwicklung der Anzahl der Erwerbspersonen und der Erwerbstätigen. Quelle: MEA, eigene Berechnungen

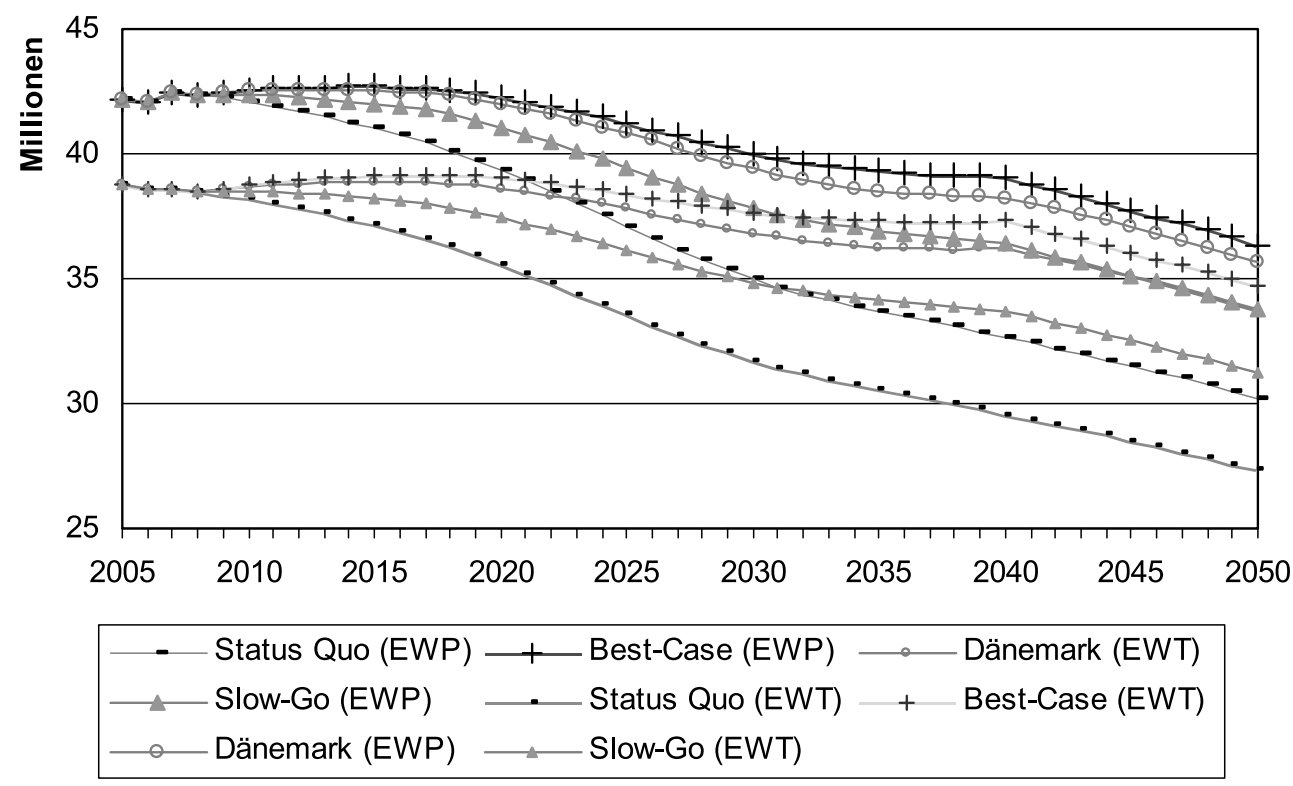

Entwicklung der Anzahl der Erwerbstätigen. Ebenso wie die Anzahl der Erwerbspersonen geht die Anzahl der Erwerbstätigen in allen vier Szenarien zurück (siehe Abb. 5). Im Dänemark-Szenario reduziert sich die Erwerbstätigenanzahl bis 2040 um über 2,5 Mio. auf etwa 36,2 Mio. Im Szenario „Best Case“ sind es 37,6 Mio., im Szenario „Slow-Go“ nur noch 33,7 Mio. im Jahr 2040. Im Status quo ergibt sich ein Rückgang von über 11 Mio. auf knappe 27,4 Mio. Erwerbstätige 2040. Letzteres entspricht einem Rückgang von fast $30 \%$.

Die Differenz zwischen der Erwerbspersonen- und der Erwerbstätigenanzahl verringert sich dabei von etwa
3,5 Mio. heute auf etwa 2,8 Mio. im Jahr 2040 im Status quo, 2,5 Mio. im Szenario „Slow-Go“ und etwa 1,7 Mio. in den Szenarien „Dänemark“ und „Best Case“. Dies ist erstens darauf zurückzuführen, dass sich die Arbeitslosenquote im Zeitverlauf auf eine immer geringere Erwerbspersonenanzahl bezieht. Zweitens verringert sich die Differenz im Dänemark- und Best-Case-Szenario zusätzlich aufgrund der annahmegemäßen Reduktion der Arbeitslosigkeit.

Reformgeschwindigkeit. Im Dänemark-Szenario gehen wir von einer Anpassung der Erwerbsquoten bis 2040 aus. Abbildung 6 zeigt die resultierende Arbeitsangebots-

Abb. 6 Verschiedene Anpassungszeiträume im DänemarkSzenario. Quelle: MEA, eigene Berechnungen, Dänemark Szenario

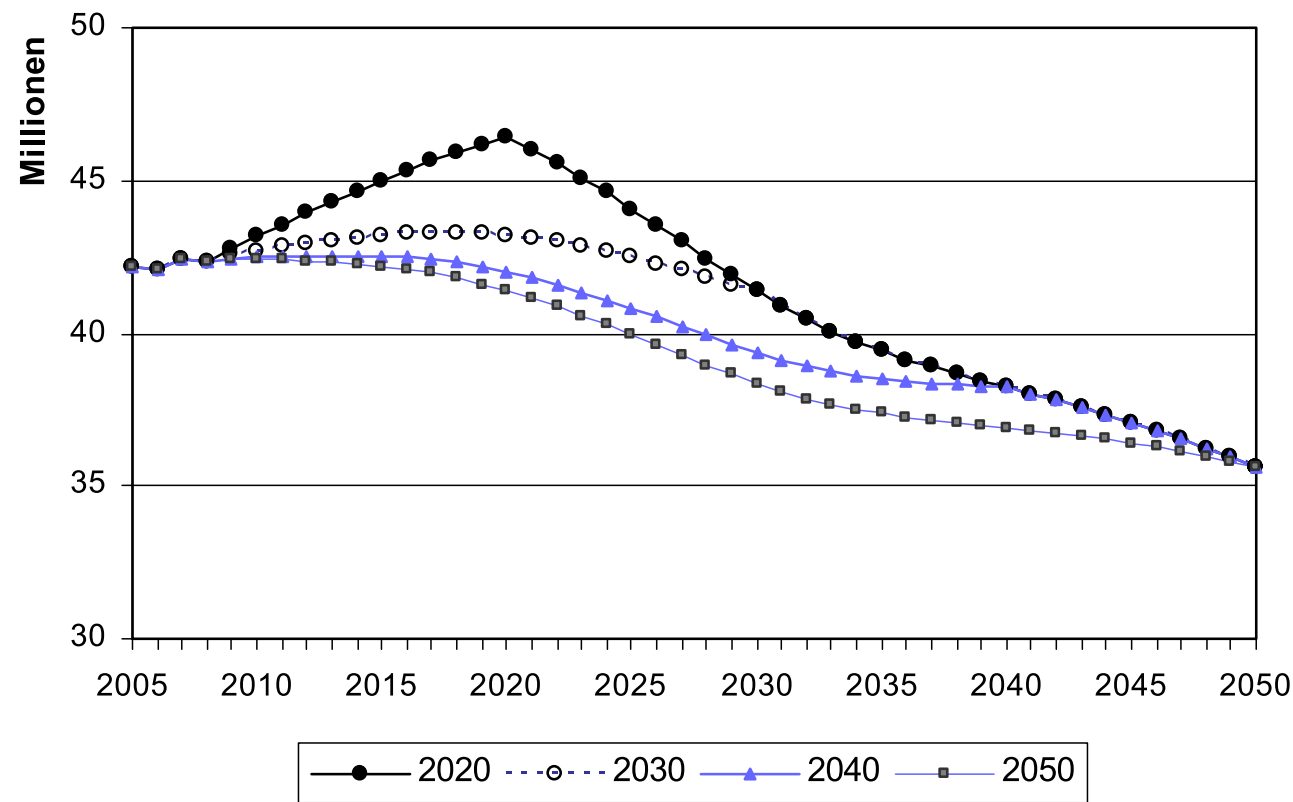


Abb. 7 Entwicklung der Anzahl der Erwerbspersonen bei alternativen Bevölkerungsprognosen. Quelle: MEA, eigene Berechnungen, Dänemark-Szenario

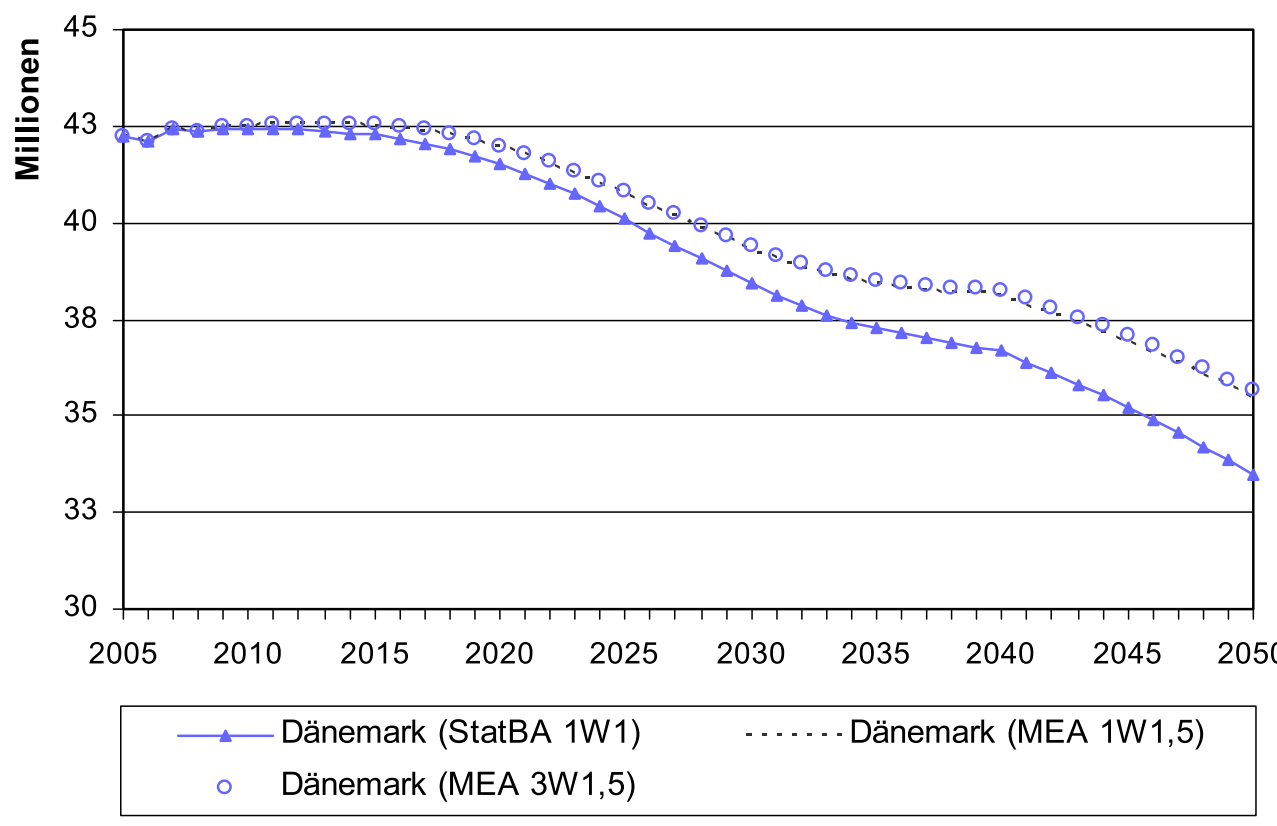

entwicklung, wenn sich diese Anpassung schneller bzw. langsamer vollzieht.

Bei einer Anpassung der deutschen Erwerbsquoten an die dänischen bereits bis zum Jahr 2020 würde die Anzahl der Erwerbspersonen auf nahezu 46,5 Mio. steigen, was einem Zuwachs von über 4 Mio. entspricht. Dieser Zuwachs begründet sich durch den starken Anstieg der Erwerbsquoten (die sich über einen kürzeren Zeitraum angleichen) bei einem noch recht verhaltenen Bevölkerungsrückgang. Allerdings ist dieser Zuwachs an Erwerbspersonen nicht nachhaltig, denn nach 2020 geht die Anzahl der Erwerbspersonen wieder zurück und zwar gemäß dem gleichen Trend wie auch in den anderen Szenariovarianten bei Konstanz der Erwerbsquoten. Hier bestimmt allein die Bevölkerung und die Alterstruktur die Entwicklung in der Anzahl der Erwerbspersonen. Bei einer Anpassung bis 2030 lässt sich dieser „Knick“ nicht mehr beobachten. Stattdessen steigt die Anzahl der Erwerbspersonen nur noch leicht auf gut 43 Mio. im Jahr 2020, sinkt bis 2030 nur knapp unter das heutige Niveau und entwickelt sich danach gemäß dem Trend. Eine langsamere Anpassung bis zum Jahr 2050 hingegen führt zwischen 2020 und $2045 \mathrm{zu}$ einer um teils über 1 Mio. niedrigeren Erwerbstätigenzahl als bei einer Anpassung bis 2040.

\section{Sensitivität bezüglich der Bevölkerungsprojektion.}

Schließlich zeigt Abb. 7 die Entwicklung der Anzahl der Erwerbspersonen im Dänemark-Szenario, wenn eine pessimistischere Bevölkerungsprojektion unterstellt wird, hier beide MEA Bevölkerungsprojektionen im Vergleich mit der Variante 1W1 der 11. koordinierten Bevölkerungsvorausberechnung.
Da sich die Bevölkerungsprojektionen MEA 3W1,5 und MEA 1W1,5 nur durch unterschiedliche Annahmen zur Lebenserwartung unterscheiden, sind die Unterschiede minimal. Anders sieht dies bei der Variante 1W1 des Statistischen Bundesamtes aus. Hier entwickelt sich die Anzahl der Erwerbspersonen im Dänemark-Szenario deutlich unterhalb der Entwicklung bei zugrunde liegenden MEA-Bevölkerungsprojektionen (etwa 1,6 Mio. im Jahr 2040). Der Grund liegt in den moderateren Migrationsannahmen (100.000 anstatt 150.000), die zu einem geringeren Erwerbspersonenpotenzial bei den Jüngeren führen. Analog dazu führt eine Nettomigration von 200.000 anstatt 150.000 zu einer um etwa 1,6 Mio. höheren Erwerbsbevölkerung 2040. Es sei angemerkt, dass selbst ein höherer Wanderungssaldo zwar die Anzahl der Erwerbspersonen generell erhöhen, ${ }^{11}$ der allgemeine Trend eines Rückgangs der Erwerbspersonenzahl jedoch auch dadurch letztlich nicht aufgehalten werden kann.

\subsection{Konsequenzen für die ökonomische Entwicklung}

Die Entwicklung der Anzahl der Erwerbstätigen, die in diesem Beitrag vorgestellt wird, ist zentral für das Verständnis der ökonomischen Auswirkungen des demografischen Wandels. Wir stellen im Folgenden Kennzahlen für zwei Auswirkungen vor, die in der öffentlichen Diskussion besonders intensiv diskutiert werden: Die Stabilität der durch Beiträge auf Arbeitseinkommen finanzierten sozialen

\footnotetext{
${ }^{11}$ Zur Rolle der Zuwanderung in der Debatte um die Auswirkungen des
} demografischen Wandels, siehe bspw. Börsch-Supan (2002). 
Abb. 8 Entwicklung des Rentnerquotienten. Quelle: MEA, eigene Berechnungen

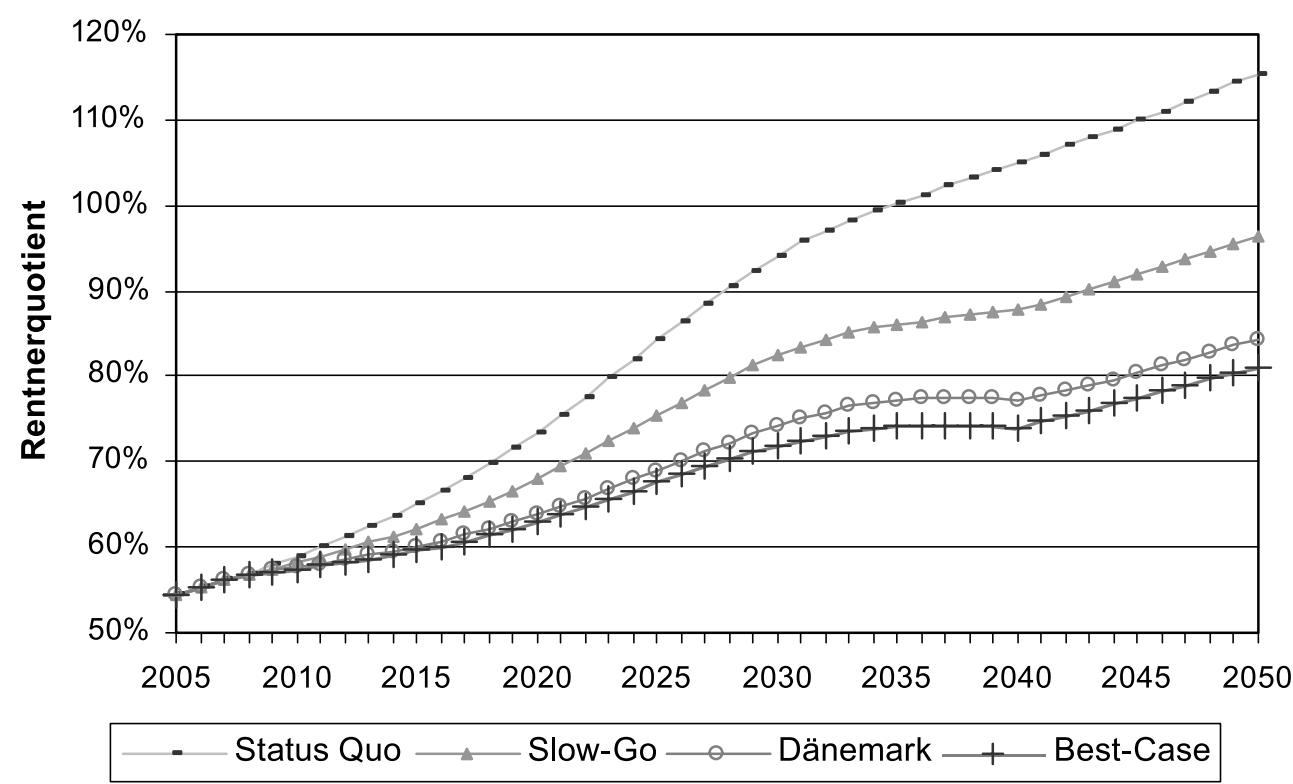

Sicherungssysteme und ein mögliches Sinken des Lebensstandards, vor allem im Vergleich mit Ländern, die weniger altern als Deutschland.

Stabilität der sozialen Sicherungssysteme. Die finanzielle Stabilität der umlagefinanzierten Sozialsysteme hängt von der Anzahl der Einzahler relativ zur Anzahl der Leistungsempfänger ab. Dieser sogenannte „Systemlastquotient“ ist am einfachsten in der Rentenversicherung als das zahlenmäßige Verhältnis von Erwerbstätigen zu Rentnern, dem sogenannten Rentnerquotienten, berechenbar. ${ }^{12}$ Ein höherer Rentenquotient impliziert höhere Beiträge und/oder niedrigere Renten; je höher der Rentnerquotient ist, desto schwieriger ist es, das Umlageverfahren aufrechtzuerhalten.

Abbildung 8 zeigt den Rentnerquotienten für die verschiedenen Arbeitsmarktszenarien. In allen Szenarien kommt es zu einem Anstieg des Rentnerquotienten. Die Spannweite zwischen den beiden Extremszenarien ist allerdings groß. ${ }^{13}$ Bei einer sehr positiven Erwerbstätigkeitsentwicklung wie im Best-Case-Szenario steigt der Rentnerquotient bis auf etwa 73,1\% im Jahr 2040 an. Im Status quo hingegen erhöht sich der Rentnerquotient bis zum Jahr 2040 auf knapp 105\%, d. h. dass 2040 in diesem Fall ein Erwerbstätiger alleine mehr als einen Rentner finanzieren muss. Im Dänemark-Szenario ist der Rentnerquotient

\footnotetext{
${ }^{12}$ Als Rentner betrachten wir in diesem Papier generell alle aus dem Arbeitsmarkt ausgeschiedenen Personen, als Erwerbstätige zählen Arbeitnehmer und Selbstständige. Der Rentnerquotient fällt hier somit niedriger aus als in der klassischen Betrachtung der Rentenversicherung, bei der die eine staatliche Rente beziehenden Personen ins Verhältnis zu den Beitragszahlern gesetzt werden.

${ }^{13}$ Dies gilt insbesondere im Vergleich zu der Variation zwischen den in Abb. 7 gezeigten Bevölkerungsprojektionen.
}

2040 um etwa 4 Prozentpunkte höher als im Best-CaseSzenario. Auch hier zeigt sich wieder, dass selbst bei einer nahezu bestmöglichen Ausschöpfung des Erwerbspersonenpotenzials der langfristige Trend nicht komplett umgangen werden kann, dass jedoch der unvermeidliche Anstieg auf einem deutlich geringeren Niveau stattfindet.

Der Druck auf die sozialen Sicherungssysteme kann somit nur teilweise über den Arbeitsmarkt abgefedert werden. Vor allem dem Anstieg in der Lebenserwartung kann nur sehr begrenzt eine höhere Erwerbsbeteiligung Älterer entgegengesetzt werden. Selbst bei äußerst pessimistischen Annahmen über die künftige Entwicklung der Lebenserwartung bleibt der Druck groß. Es ist davon auszugehen, dass der Rentnerquotient innerhalb der nächsten 30 Jahre auch im bestmöglichen Fall (Szenario „Best Case“ basierend auf MEA 1W1,5) um mindestens 15 Prozentpunkte ansteigen wird. Dies bedeutet für die aktive erwerbstätige Bevölkerung eine zusätzliche Belastung allein bis 2040 von mehr als einem Viertel.

Entwicklung des Lebensstandards. Abbildung 8 zeigt auch, dass die Anzahl der Erwerbstätigen nicht nur absolut, sondern auch relativ zur Bevölkerung sinken wird. Mit einem geringeren Arbeitseinsatz pro Kopf der Bevölkerung können wir jedoch nicht ohne weiteres unseren gewohnten Lebensstandard halten. Der relative Rückgang in der Anzahl der Erwerbstätigen ist allerdings geringer als der absolute Rückgang. Daher zeigen wir in Abb. 9 die sogenannte „Stützquote“, die als der Anteil der Erwerbstätigen an der erwachsenen Bevölkerung ab dem Alter 15 definiert ist. Dies entspricht im Großen und Ganzen der Anzahl der Konsumenten, deren Nachfrage nach Gütern und Dienstleistungen von den Erwerbstätigen produziert werden muss. 
Abb. 9 Entwicklung der Stützquote. Quelle: MEA, eigene Berechnungen

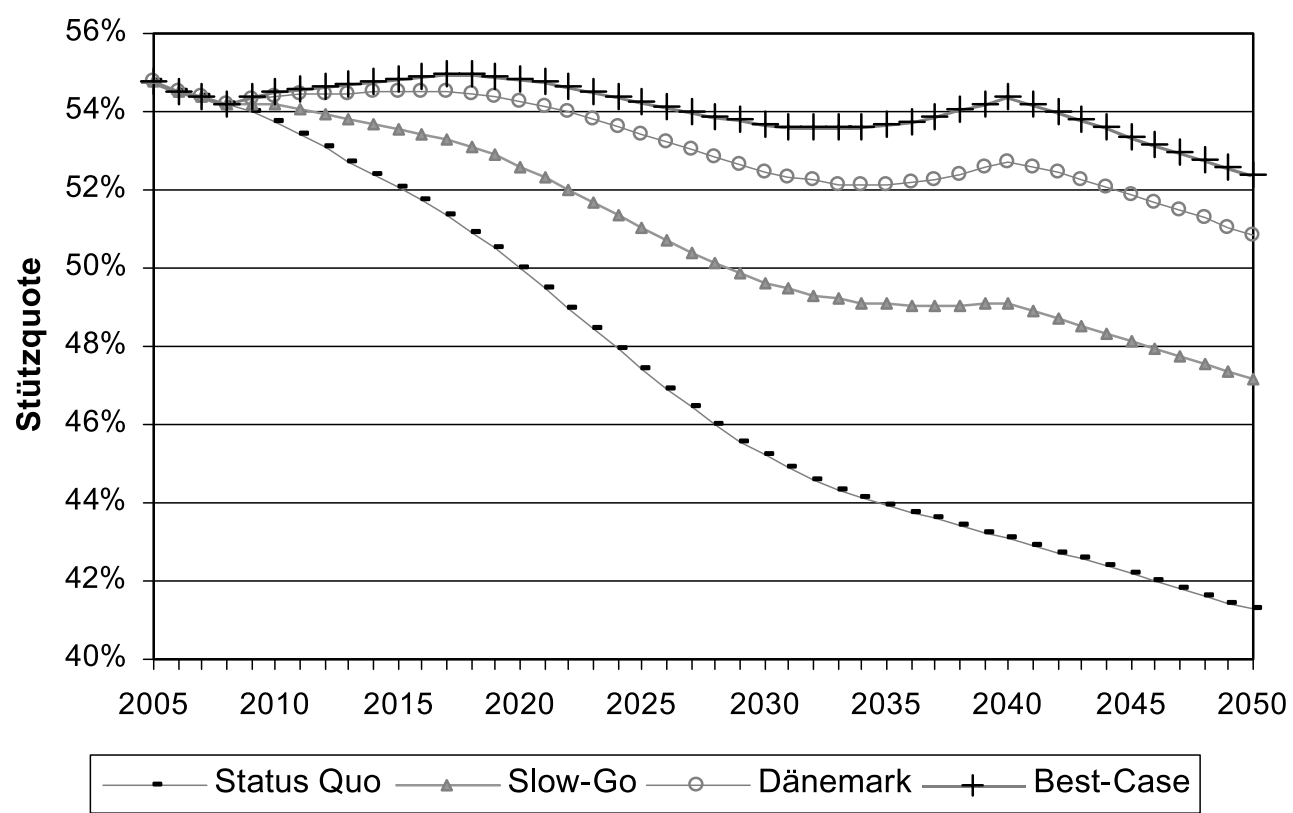

Mit Ausnahme des Status-Quo-Szenarios beginnt der wesentliche Rückgang hier erst ab 2015. Für das Dänemark-Szenario fällt die Stützquote von heute 54,8 auf 52,1\% im Jahr 2035. Danach steigt die Stützquote bis 2040 wieder an. Der erneute Anstieg hängt mit den unterstellten Anpassungen am Arbeitsmarkt zusammen, die aufgrund der Altersstruktur die Anzahl der Erwerbstätigen nach 2035 schneller wachsen lassen als die Zunahme in der Lebenserwartung die erwachsene Gesamtbevölkerung wachsen lässt. Nach 2040 geht die Stützquote bei konstanten Erwerbstätigenquoten und sinkenden Bevölkerungszahlen weiter zurück. Für das Status-Quo-Szenario, in dem keine Anpassungen am Arbeitsmarkt angenommen werden, ist die Stützquote im gesamten Zeitraum steil und streng monoton fallend. Als ökonomische Konsequenz ergibt sich, dass sich zukünftige Erhöhungen des Lebensstandards allein aus vermehrtem Kapitaleinsatz oder Steigerungen der Gesamtfaktorproduktivität speisen müssen. Dies wird nicht einfach sein, da sich auch die Altersstruktur der Erwerbstätigen deutlich ändern wird.

Altersstruktur. Abbildung 10 zeigt die Altersverteilung der Erwerbspersonen im Dänemark-Szenario für die Jahre 2010,
Abb. 10 Altersstruktur der Erwerbspersonen. Quelle: MEA, eigene Berechnungen, Dänemark-Szenario

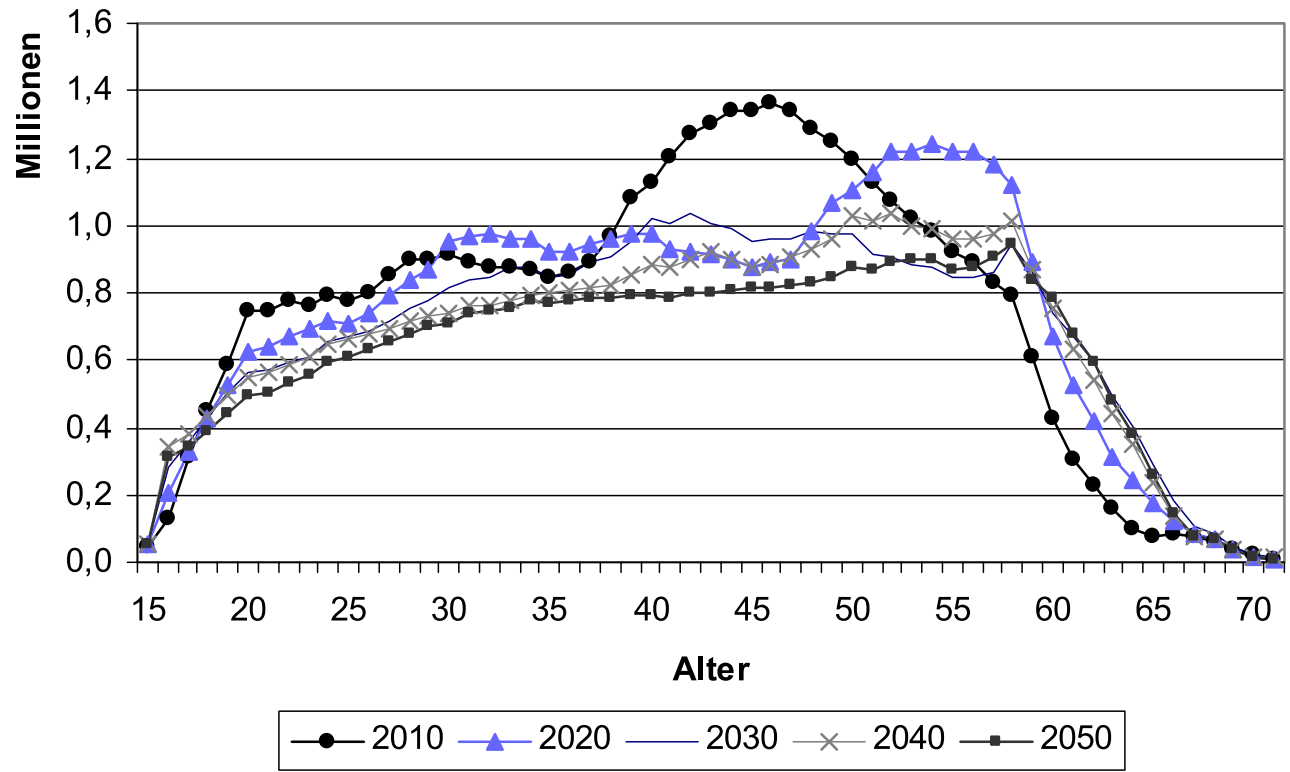


Abb. 11 Anteil der Erwerbspersonen im Alter 55+. Quelle: MEA, eigene Berechnungen

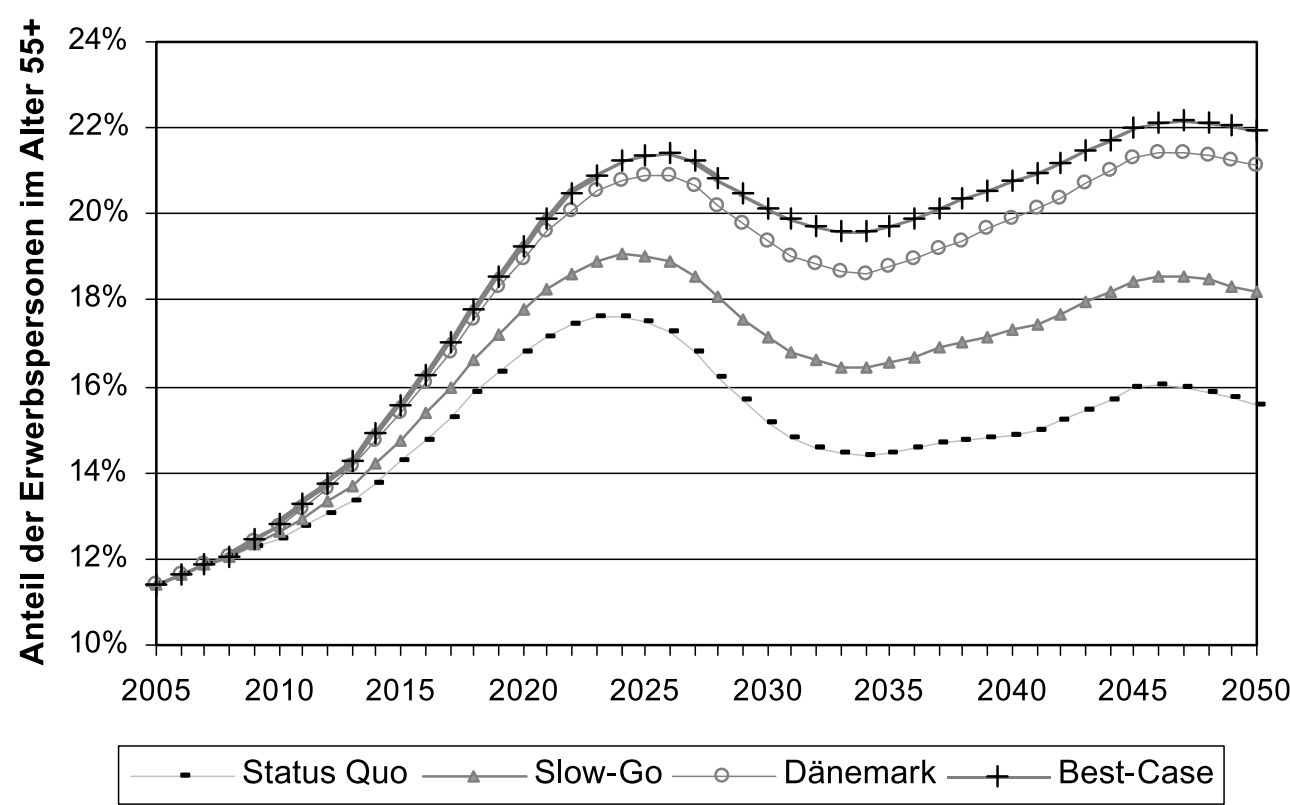

2020, 2030, 2040 und 2050. Man sieht deutlich, wie der Gipfel der Verteilung sich zunächst nach rechts bewegt und dann zwischen 2020 und 2030, wenn die Baby-Boomer in Rente gehen, verschwindet. Danach wird die Altersverteilung flacher und verändert sich in den Folgejahren nur noch wenig.

Das durchschnittliche Alter der Erwerbspersonen wird im Dänemark-Szenario in den nächsten 20 Jahren von 39,8 auf etwa 41,7 im Jahr 2025 ansteigen, dann für 10 Jahre lang wieder leicht rückläufig sein und schließlich bis 2050 um ein weiteres Vierteljahr auf etwa 42 Jahre ansteigen. Im BestCase-Szenario ist die Entwicklung ähnlich. In den Szenarien Slow-Go und Status quo hingegen fällt der Rückgang des Durchschnittsalters nach 2025 wesentlich stärker aus, weil dann der Großteil der Baby-Boom-Generation bereits in Rente gegangen sein wird. Der Altersstrukturwandel wird noch deutlicher, wenn man den Anteil der Erwerbspersonen im Alter 55+ betrachtet (Abb. 11). Der Anteil älterer Erwerbspersonen wird sich bis zum Jahr 2025 von derzeit 11 auf knapp 22\% im Dänemark-Szenario und im Best-CaseSzenario fast verdoppeln. Im Szenario Slow-Go nimmt der Anteil dieser Erwerbspersonen immerhin immer noch um fast zwei Drittel zu.

Danach sieht man die eine ,Verjüngung“ der Erwerbspersonen, weil dann die Babyboomgeneration in Rente gehen wird. Dieser Effekt ist jedoch nur temporär. Die Altersstrukturverschiebung ist also kein Übergangsphänomen, sondern eine permanente Veränderung. Der Vergleich der Szenarien zeigt auch, dass wir uns in jedem Fall auf eine dramatische Alterstrukturverschiebung der Beschäftigten einstellen müssen.

\section{Wirkungsanalyse der Arbeitsangebotsparameter}

Den vier im vorangegangenen Abschnitt vorgestellten Szenarien liegen Kombinationen von unterschiedlichen Annahmen bezüglich der das Arbeitsangebot bestimmenden Parameter (Berufseintrittsalter, Frauenerwerbstätigkeit und Renteneintrittsalter) zugrunde. In diesem Abschnitt zeigen wir, wie groß der Einfluss jedes einzelnen dieser Parameter auf das Arbeitsangebot ist.

Abbildung 12 zeigt, welche Auswirkung eine Herabsetzung des Berufseintrittsalters (BEA) um ein Jahr bzw. zwei Jahre bis 2040 auf die Erwerbsquoten und damit die Anzahl der Erwerbspersonen am Arbeitsmarkt hat. Die Anzahl der Erwerbspersonen im Status quo wird dazu auf Eins normiert.

Eine Herabsetzung des Berufseintrittsalters um ein Jahr bis 2040 bewirkt demnach eine Steigerung der Anzahl der

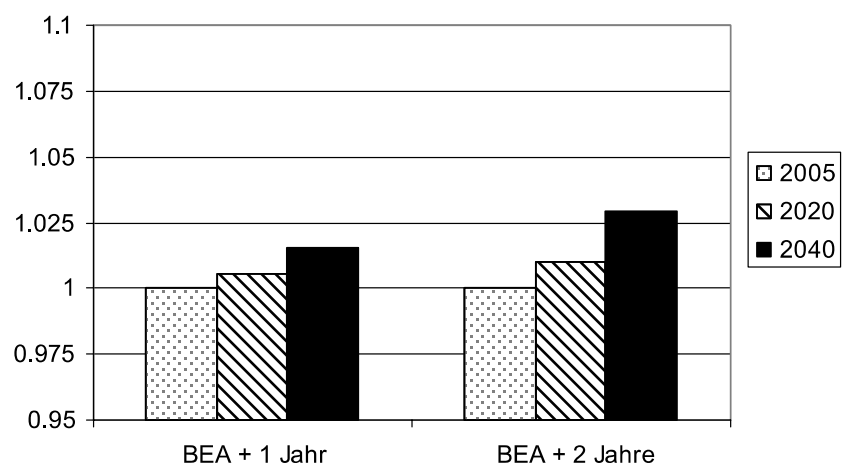

Abb. 12 Berufseintrittsalter und Erwerbspersonenanzahl. Quelle: MEA, eigene Berechnungen 
Erwerbspersonen um 0,7\% bis zum Jahr 2020 und 1,5\% bis $2040 \mathrm{im}$ Vergleich zum Status quo. Wird das Berufseintrittsalter um zwei Jahre nach vorne verschoben, so verdoppelt sich der Effekt auf 1,4\% bis zum Jahr 2020 und 3,0\% bis 2040.

Im Hinblick auf die Diskussion um kürzere Ausbildungszeiten ist ein um ein Jahr nach vorne verschobener Berufseintritt künftig sicherlich realistisch. Eine Verschiebung des Berufseintrittsalters um mehr als zwei Jahre nach vorne hingegen erscheint unplausibel, da die Ausbildungszeiten dann unter die kürzesten in den EU-Mitgliedsländern fallen würden. Die Auswirkungen auf die Erwerbspersonenanzahl sind insgesamt eher gering, da die Jahrgangsstärken niedrig sind. Allerdings ist zu berücksichtigen, dass ein früherer Einstieg der Jüngeren in den Arbeitsmarkt die Altersstruktur der Erwerbspersonen verjüngt.

Erhöhung der Frauenerwerbsquote. Eine Anpassung der Frauenerwerbsquote (FEWT) an die der Männer hingegen kann sehr große Auswirkungen auf die Anzahl der Erwerbspersonen haben, wie Abb. 13 zeigt. Demnach bewirkt eine Anpassung der Frauenerwerbstätigkeit an die der Männer um $20 \%$ bis 2040 bereits eine Erhöhung der Anzahl der Erwerbspersonen um $1 \%$ bis 2020 und $2 \%$ bis 2040 . Eine größere Anpassung um 40, 60 oder 80\% der Differenz zwischen den Erwerbsquoten der Frauen und Männer führt entsprechend zu einer Erhöhung der Erwerbspersonenanzahl um 2, 3 und $4 \%$ bis 2020 und 4, 6 und $8 \%$ bis 2040 . Ebenso wie beim Berufseintritt sind die Auswirkungen der einzelnen Änderungen somit im Wesentlichen proportional.

Eine Angleichung der Frauenerwerbsquoten an die der Männer hat somit eine relative große Wirkung. Da Deutschland im Vergleich mit anderen Ländern wie bspw. Dänemark derzeit noch eine eher geringe Erwerbsbeteiligung von Frauen verzeichnet (vgl. Abb. 5), besteht hier noch Spielraum für künftige Anpassungen. Voraussetzung ist allerdings eine entsprechende Politik, die die Vereinbarkeit von Familie und Beruf weiter fördert und Abschied von den klassischen Geschlechterrollen nimmt.

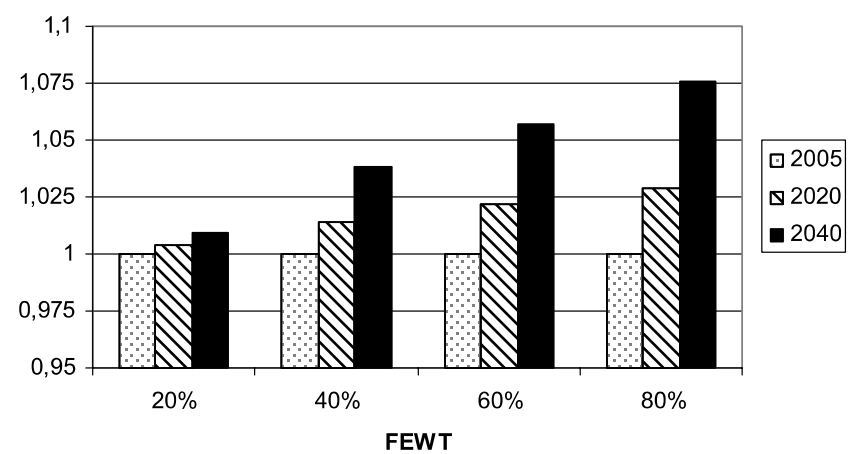

Abb. 13 Frauenerwerbstätigkeit und Erwerbspersonenanzahl. Quelle: MEA, eigene Berechnungen

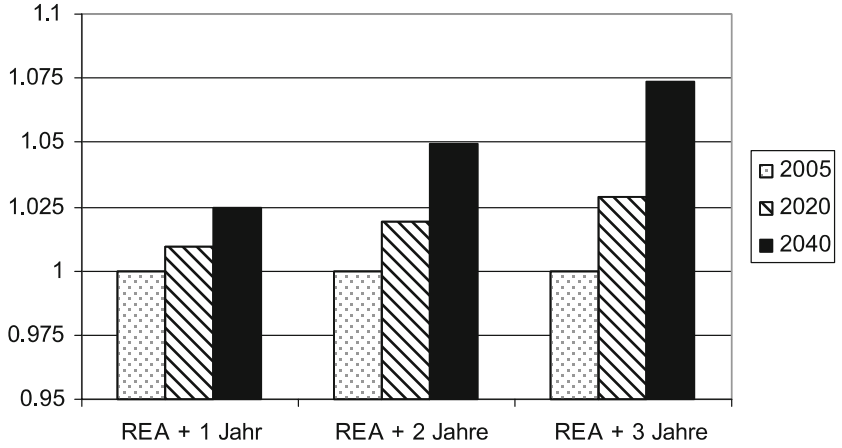

Abb. 14 Renteneintrittsalter und Erwerbspersonenanzahl. Quelle: MEA, eigene Berechnungen

Erhöhung des Renteneintrittsalters. Eine Erhöhung des effektiven Renteneintrittsalters (REA) hat ebenfalls eine große Wirkung auf das Arbeitsangebot, siehe Abb. 14. Eine Erhöhung um ein Jahr bis 2040 führt zu einer Erhöhung der Erwerbspersonenzahl um 1,2\% bis 2020 und 2,4\% bis 2040. Bei einer Erhöhung um zwei bzw. drei Jahre steigt die Anzahl der Erwerbspersonen wiederum in etwa proportional.

Aufgrund der Abschaffung eines Großteils der Frühverrentungswege im Rahmen der 1998er-Rentenreform ${ }^{14}$ hat sich das durchschnittliche Renteneintrittsalter für Altersrenten innerhalb der letzten Dekade bereits von 61,2 Jahren im Jahr 1995 auf 62,7 Jahre im Jahr 2003 erhöht. ${ }^{15}$ Berücksichtigt man Erwerbsminderungsrenten (ab 50 Jahre), so ist das Zugangsalter niedriger (62,3 Jahre im Jahr 2005), der Anstieg des effektiven Renteneintrittsalters seit 1996 war aber stärker (siehe Brussig u. Wojtkowski 2006). Im Rahmen dieser Entwicklung ist künftig ein weiterer Anstieg um ein Jahr vorstellbar, bis die Übergangsregelungen im Jahr 2015 komplett ausgelaufen sind. Eine darüber hinaus gehende Erhöhung des mittleren Renteneintrittsalters um zwei oder gar drei Jahre baut dann auf der Erhöhung des Regelrentenalters von 65 auf 67 Jahre auf (siehe hierzu Clemens 2006 und Bucher-Koenen u. Wilke 2007).

Vergleich der Hebelwirkungen. Abbildung 15 zeigt schließlich das Wirkungsausmaß der verschiedenen Parameter im Vergleich. ${ }^{16}$ Dabei wird von einer Herabsetzung

\footnotetext{
14 Für einen Überblick über den Rentenreformprozess in Deutschland siehe Börsch-Supan u. Wilke (2003).

${ }^{15}$ Siehe Berkel u. Börsch-Supan (2003). Für eine Diskussion der Wirkung von Frühverrentungsoptionen auf die Wahl des Renteneintrittsalters siehe auch Schmidt (1995) sowie Riphahn u. Schmidt (1997) und Siddiqui (1997).

${ }^{16}$ Üblicherweise werden in der ökonomischen Analyse solche Vergleiche mithilfe von Elastizitäten durchgeführt, da so die Wirkungen prozentual normiert werden. In diesem Fall ist dies weniger hilfreich, da nicht klar ist, welche Basis zu verwenden ist. Eine Erhöhung des Renteneintrittsalters
} 


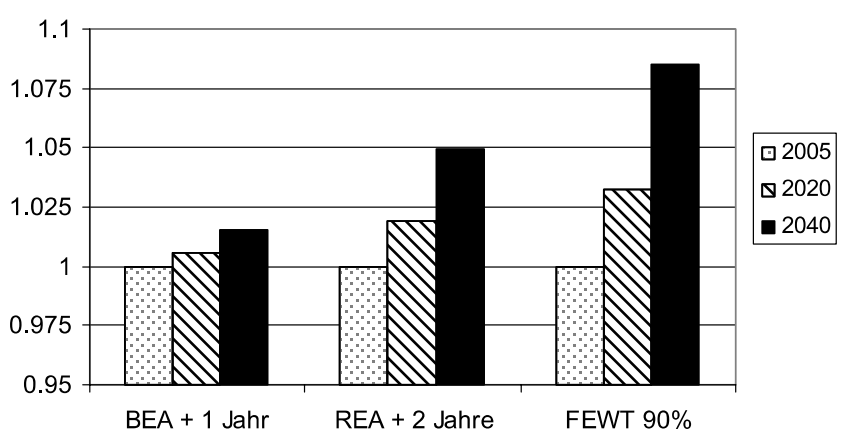

Abb. 15 Wirkungsausmaß der verschiedenen Stellgrößen. Quelle: MEA, eigene Berechnungen

des Berufseintrittsalters um ein Jahr, einer Anpassung der Frauenerwerbstätigkeit an die Männer um $90 \%$ und einer Erhöhung des Renteneintrittsalters um zwei Jahre ausgegangen. Diese Annahmen entsprechen zusammengenommen in etwa dem Dänemark-Szenario.

Die Herabsetzung des Berufseintrittsalters um ein Jahr erhöht die Anzahl der Erwerbspersonen 2040 um 1,6\%, eine höhere Frauenerwerbstätigkeit um $8,6 \%$ und ein um zwei Jahre späteres Renteneintrittsalter um $4,9 \%$. Addiert man diese Teileffekte zusammen, so ergibt sich ein Gesamteffekt von 15,1\% im Jahr 2040. Der Gesamteffekt einer Kombination ist allerdings höher (Abb. 5). Dies liegt an der verstärkenden Interaktion der einzelnen Parameterveränderungen, da bspw. eine Erhöhung des mittleren Renteneintrittsalters auch auf den erhöhten Frauenanteil zutrifft.

\section{Fazit und Ausblick}

Dieser Beitrag zeigt, welche Arbeitsangebots- und Erwerbstätigenentwicklung für Deutschland bis zum Jahr 2050 erwartet werden kann. Sie hängt maßgeblich davon $a b$, wie gut es gelingt, durch Strukturreformen entsprechende Veränderungen des Arbeitsangebotsverhaltens und der Arbeitsnachfrage einzuleiten. Ausmaß und Geschwindigkeit solcher Strukturreformen werden daher szenarisch modelliert.

Spätestens nach 2020 wird sowohl die Anzahl der Erwerbspersonen als auch die Anzahl der Erwerbstätigen aufgrund des Bevölkerungsrückgangs spürbar zurückgehen. Weder eine bestmögliche Ausnutzung des Erwerbspersonenpotenzials noch eine realistische Zunahme der Migration können diesen Trend vollständig verhindern.

Im Dänemark-Szenario geht die Anzahl an Erwerbspersonen etwa ab 2015 langsam zurück. Den gleichen Trend für Deutschland findet auch das Institut für Arbeitsmarkt- und

um ein Jahr bspw. auf die gesamte Lebenszeit zu beziehen, erscheint nicht sinnvoll.
Berufsforschung (IAB) in seinen Arbeitsmarktprojektionen und Langfristberechnungen zum Erwerbspersonenpotenzial (siehe Fuchs u. Dörfler 2005a, b sowie Fuchs u. Söhnlein 2007) sowie die Europäische Kommission (siehe Carone 2005), obwohl sich die Prognosen im Detail aufgrund unterschiedlicher Bevölkerungs- und Erwerbstätigkeitsannahmen unterscheiden. Eine wichtige Einsicht ist, dass sich das Niveau dieses Rückgangs in den Arbeitsmarktreformszenarien deutlich im Vergleich zum Status quo mindern lässt.

Auch ein Vergleich der sich im Dänemark-Szenario ergebenden absoluten Erwerbstätigenzahlen und des Rentnerquotienten mit der Erwerbstätigenprognose der RürupKommission (vgl. Kommission für die Nachhaltigkeit in der Finanzierung der Sozialen Sicherungssysteme 2003) zeigt eine große Übereinstimmung. Dies ist insofern überraschend, als die Erwerbstätigenprognose der Kommission erstens auf einer bis 2050 wesentlich optimistischeren Bevölkerungsprognose basiert (vgl. „Rürup“ in Abb. 1), und sie zweitens neben dem Arbeitsangebot auch explizit die Entwicklung der künftigen Arbeitsnachfrage berücksichtigt, wovon in diesem Papier abgesehen wurde. Diese Koinzidenz veranschaulicht, wie komplex der Einfluss unterschiedlicher Prognoseannahmen auf die Ergebnisse ist und dass abweichende Bevölkerungs- und Arbeitsmarktannahmen sich in ihrer Wirkung gegenseitig aufheben können.

Schließlich zeigt sich, dass innerhalb einer realistischen Szenarienspannbreite die Ergebnisse recht robust sind: Welche künftige Entwicklung auch immer letztlich realisiert wird, sie kann keine Überraschung sein, denn die natürlichen Ober- und Untergrenzen sind bekannt (,Status quo“ und „Best Case“).

Wirtschaftspolitische Implikationen. Unsere Wirkungsanalyse der Einzelparameter zeigte, dass neben kürzeren Ausbildungszeiten und einem damit einhergehenden niedrigeren mittleren Berufseintrittsalter insbesondere eine Erhöhung der Frauenerwerbstätigkeit und eine Heraufsetzung des Renteneintrittsalters große Auswirkungen auf das Arbeitsangebot haben.

In Bezug auf die Frauenerwerbstätigkeit müssen in Deutschland vor allem Voraussetzungen für eine bessere Vereinbarkeit von Familie und Beruf geschaffen werden (vgl. Wissenschaftlicher Beirat 2005). So ist die Erwerbsbeteiligung kinderloser Frauen in Deutschland im internationalen Vergleich gut; erst wenn Frauen mit Kindern betrachtet werden, fällt Deutschland im Ranking zurück (siehe Dressel et al. 2005).

Eine Heraufsetzung des Renteneintrittsalters wirkt sich nicht nur positiv auf die Anzahl der Erwerbspersonen, sondern ebenso günstig auf die Anzahl der Rentner aus und trägt so zu einem geringeren Anstieg des Rentnerquotien- 
ten bei. Neben einem aufgrund der vergangenen Rentenreformen 1992, 2001 und $2004 \mathrm{zu}$ erwartenden weiteren Anstieg des mittleren Renteneintrittsalter lässt sich ein weiterer Anstieg durch eine Anhebung des gesetzlichen Rentenalters auf 67 Jahre erreichen.

Schließlich ist das Arbeitsvolumen insgesamt, also das die tatsächlich geleistete Arbeitszeit der Erwerbspersonen (in Stunden), von großer Bedeutung. Der Beschäftigungszuwachs der Frauen in den 1990er-Jahren in Deutschland basiert bspw. nahezu vollständig auf Teilzeitarbeitzuwächsen (siehe Bothfelt et al. 2005 sowie Dressel 2005). Insgesamt ist das Arbeitsvolumen aufgrund der geringeren Wochenarbeitszeit, vermehrten Urlaubsansprüchen, der im Vergleich zu früher geringen Erwerbsbeteiligung der Älteren und nicht zuletzt der hohen Arbeitslosigkeit in den vergangenen Dekaden jedoch stark zurückgegangen (siehe Börsch-Supan 2000). Es gibt jedoch einige Indizien dafür, dass das Arbeitsvolumen im Zuge der erwarteten Annäherung der Erwerbsmuster von Frauen und Männern künftig wieder etwas ansteigen wird (siehe z. B. Allmendinger u. Ebner 2005). So lassen sich bspw. im europäischen Vergleich umso niedrigere Arbeitszeitdifferenzen zwischen Männern und Frauen ausmachen, je besser die vorhandene Infrastruktur von Kindererziehungsleistungen ist (siehe hierzu auch European Commission 2000). Dies würde die finanzielle Lage der Sozialversicherungen zumindest mittelfristig günstig beeinflussen.

Erforderlicher Produktivitätszuwachs. Schließlich spielt neben der in diesem Beitrag quantitativ skizzierten Entwicklung auch die qualitative Entwicklung auf dem Arbeitsmarkt eine wichtige Rolle, denn der Rückgang der Stützquote bedeutet, dass sich zukünftige Erhöhungen des Lebensstandards allein aus vermehrtem Kapitaleinsatz oder Steigerungen der Gesamtfaktorproduktivität speisen müssen. Da eine erhöhte Sparquote einer alternden Bevölkerung unwahrscheinlich ist, wird Deutschland in Zukunft noch mehr auf Produktivitätszuwächse angewiesen sein.

Die folgende Überschlagsrechnung zeigt die Größenordnungen. Der relative Rückgang in der Anzahl der Erwerbstätigen führt allein bis 2035 dazu, dass im Dänemark-Szenario die Erwerbstätigen im Jahre 2035 nahezu 5\% mehr leisten müssten als heute, um die gleiche Menge an Konsum- und Investitionsgütern pro Kopf zu produzieren. Dies entspräche einem jährlichen Produktivitätszuwachs von etwa 0,2 Prozentpunkten bis 2035, zusätzlich zum historisch normalen Produktivitätszuwachs von etwa $1,4 \%{ }^{17}$ pro Jahr. Ein jährlicher Produktivitätszuwachs von 1,6\% ist nicht unmöglich, in vielen Ländern sogar normal; er würde den Lebens-

\footnotetext{
${ }^{17}$ Siehe Buchheim (1998). Das Institut für Arbeitsmarkt- und Berufsforschung (IAB) rechnet in seiner Langfristprojektion des Arbeitskräftebedarfs bis 2020 sogar nur mit 1,3\%, siehe Schnur u. Zika (2005).
}

standard relativ zu unseren EU-Nachbarn in etwa stabil halten.

Im Status-quo-Szenario müssten die Erwerbstätigen im Jahr 2035 hingegen schon fast 20\% mehr leisten als heute. Dies entspräche einem jährlichen Produktivitätszuwachs von etwa 0,63 Prozentpunkten bis 2035. Eine Steigerung des heutigen Produktivitätsfortschritts auf über $2 \%$ pro Jahr für die gesamte mittelfristige Zukunft ist nur schwer vorstellbar. Das Status-Quo-Szenario zeigt daher die Größenordnung der Einbuße unseres gewohnten Lebensstandards relativ zu unseren Nachbarn, wenn sich die Erwerbstätigkeit nicht an die veränderten demografischen Verhältnisse anpasst.

Ein stärkeres Produktivitätswachstum impliziert einen weiter steigenden Bedarf an Höherqualifizierten bei gleichzeitig schrumpfender Anzahl jüngerer Erwerbspersonen. Bei einem Stillstand der Bildungsentwicklung wird es daher zu einem noch deutlicheren Fachkräftemangel als heute kommen (siehe hierzu Reinberg u. Hummel 2003). Gleichzeitig bestehen bereits heute teils massive Beschäftigungsprobleme wettbewerbsschwacher Arbeitnehmer. Eine entsprechende Bildungs- und Weiterbildungspolitik ist wichtig, um eine solche Fehlentwicklung auf dem Arbeitsmarkt zu verhindern. Der Akkumulation von Humanvermögen durch Aus- und Weiterbildung wird künftig daher ein immer höherer Stellenwert zukommen.

Insgesamt hat dieser Beitrag gezeigt, dass eine bessere Erschließung des Erwerbspersonenpotenzials die Auswirkungen der Bevölkerungsalterung auf die deutsche Volkswirtschaft deutlich abfedern kann. Auch der Druck auf die sozialen Sicherungssysteme kann durch eine maßvolle und graduelle Reformpolitik, wie sie im Dänemark-Szenario modellhaft abgebildet wird, signifikant abgemildert werden. Ein Ende des derzeitigen Reformprozesses hätte, wie die alternativen Szenarien aufzeigen, dagegen langfristig restriktive Folgen für den Lebensstandard und die finanzielle Basis unserer Sozialsysteme. Der Unterschied zwischen dem Reformszenario „Dänemark“ und dem pessimistischeren Szenario einer nur unvollständig realisierten Agenda 2010 („Slow-Go“) beträgt 2,1 Mio. Erwerbstätige im Jahr 2030. Deren Arbeitskraft ist unverzichtbar für unseren Lebensstandard und unsere Sozialversicherungen.

\section{Kurzfassung}

Der demografische Wandel führt dazu, dass es immer mehr ältere und immer weniger jüngere Menschen gibt. Dies hat nicht nur sozialpolitische Konsequenzen, wie sie im Hinblick auf die Nachhaltigkeit der sozialen Sicherungssysteme in den vergangenen Jahren in der Öffentlichkeit verstärkt diskutiert wurden, sondern beeinflusst unser gesamtes Wirtschaftsgeschehen und wird in den kommen- 
den Dekaden einen tiefgreifenden makroökonomischen Strukturwandel hervorrufen. Maßgeblich für das Ausmaß dieser sich abzeichnenden Entwicklung ist jedoch nicht nur das demografisch-zahlenmäßige Verhältnis der Älteren zu den Jüngeren in einer Gesellschaft. Für die ökonomischen Konsequenzen ist insbesondere die Beteiligung am Erwerbsleben wichtig. Dazu bedarf es Projektionen zur mittel- und langfristigen Entwicklung der Anzahl der Erwerbspersonen sowie der Erwerbstätigen in Deutschland. Dies ist das Thema dieses Beitrags.

Fundament einer Projektion der Anzahl der Erwerbspersonen bzw. Erwerbstätigen ist zunächst die Demografie. Dieser Beitrag betont, dass die offiziellen Annahmen über die zukünftige Lebenserwartung zu pessimistisch sind, d. h. eine zu kurze Lebenszeit vorhersagen, wenn man sich an der vergangenen Entwicklung und den neuesten Ergebnissen der internationalen demografischen Forschung orientiert. Wir stellen daher zunächst verschiedene Szenarien der demografischen Entwicklung vor und wählen dasjenige, das uns am wahrscheinlichsten erscheint.

Die Entwicklung der Anzahl der Erwerbspersonen bzw. Erwerbstätigen wird nach der Demografie von den künftigen alters- und geschlechtsspezifischen Erwerbsquoten bestimmt. Diese hängen stark von zukünftigen Politikentscheidungen und den Verhaltensreaktionen auf sie ab. Sie sind kaum vorherzusagen. Wir gehen daher auch hier szenarienbasiert vor.

Wir wählen vier Szenarien, welche die Spannbreite möglicher künftiger Entwicklungen aufzeigen. Nach oben hin begrenzt die durch die Demografie vorgegebene Bevölkerung im erwerbsfähigen Alter die Entwicklungsmöglichkeiten. In unserem optimistischen Szenario gehen wir davon aus, dass dieses Erwerbspersonenpotenzial bestmöglich ausgeschöpft wird. Die Untergrenze wird durch den Status quo markiert, d.h. die Annahme, dass sich die heutigen Erwerbsquoten auch in Zukunft nicht ändern werden. Ein Rückwärtstrend der Erwerbsquoten erscheint uns hingegen nicht plausibel.

Um ein realistisches Szenario innerhalb dieser Ober- und Untergrenzen zu erhalten, und um abzuschätzen, wie weit das Erwerbspersonenpotenzial in realistischem Maße ausgeschöpft werden kann, ist ein Blick auf die europäischen Nachbarländer hilfreich, in denen ähnliche Arbeitsmarktreformen wie die deutschen stattgefunden haben. Wir wählen Dänemark als einen Referenzpunkt mit einer relativ hohen Erwerbsbeteiligung sowohl der Frauen als auch der Älteren aus. Unterstellt man einen Anstieg der deutschen Erwerbsquoten auf das dänische Niveau, so zeigt sich, dass ein Großteil des demografischen Drucks durch den Arbeitsmarkt abgefedert werden kann. Dies ist eine wichtige wirtschaftspolitische Botschaft.

Natürlich sind weitere Szenarien denkbar, vor allem ein Nachlassen der Reformtätigkeit, die jedoch nicht im voll- ständigen Stillstand unseres Status-quo-Szenarios endet. Wir modellieren dies mithilfe eines Szenarios, das wir „Slow-Go“ nennen. Dieses Szenario geht davon aus, dass die in der Agenda 2010 enthaltenen Reformen nur teilweise umgesetzt werden. Weitere Szenarien kann der Leser approximativ durch Interpolation unserer Ergebnisse bilden.

Unser Hauptergebnis ist, dass in jedem Fall, auch im günstigsten, sowohl die Erwerbspersonen- als auch die Erwerbstätigenanzahl künftig sinken wird. Spätestens nach 2020 wird ein Rückgang allein aufgrund der Bevölkerungsentwicklung erkennbar werden. Weder eine bestmögliche Ausnutzung des Erwerbspersonenpotenzials noch eine realistische Zunahme der Migration können diesen Trend vollständig verhindern. Im Dänemark-Szenario ergibt sich ein Rückgang in der Anzahl der Erwerbspersonen um etwa 2,8 auf etwa 39,4 Mio. im Jahr 2040. Ohne weitere Arbeitsmarktreformen würde die Anzahl der Erwerbspersonen bis 2040 um über 9 auf 32,6 Mio. zurückgehen. Für die Zahl der Erwerbstätigen ergibt sich ein moderaterer Rückgang von 2,5 auf 36,2 Mio. im Jahr 2040 im Dänemark-Szenario und ein wesentlich stärkerer Rückgang von 11 auf 27,4 Mio. im Status-quo-Szenario. Eine wichtige Einsicht ist, dass sich das Niveau dieses Rückgangs in den Arbeitsmarktreformszenarien im Vergleich zum Status quo deutlich mindern lässt.

Zugleich altert die Erwerbsbevölkerung. Das Durchschnittsalter wird bis zum Jahr 2040 von 40 auf knapp 42 Jahre ansteigen. Der Anteil der Erwerbspersonen im Alter von mindestens 55 Jahren wird von etwa 11 auf $20 \%$ im Jahr 2040 steigen.

Innerhalb einer realistischen Szenarienspannbreite sind unsere Ergebnisse sehr robust: Welche künftige Entwicklung auch immer letztlich realisiert wird, sie kann keine Überraschung sein, denn die natürlichen Ober- und Untergrenzen sind bekannt (,Status quo“ und „Best Case“). Unsere Wirkungsanalyse der Einzelparameter macht deutlich, dass neben kürzeren Ausbildungszeiten und einem damit einhergehenden niedrigeren mittleren Berufseintrittsalter insbesondere eine Erhöhung der Frauenerwerbstätigkeit und eine Heraufsetzung des Renteneintrittsalters große Auswirkungen auf das Arbeitsangebot haben.

Insgesamt zeigt dieser Beitrag daher, dass eine bessere Erschließung des Erwerbspersonenpotenzials die Auswirkungen der Bevölkerungsalterung auf die deutsche Volkswirtschaft deutlich abfedern kann. Auch der Druck auf die sozialen Sicherungssysteme kann durch eine maßvolle und graduelle Reformpolitik, wie sie im Dänemark-Szenario modellhaft abgebildet wird, signifikant abgemildert werden. Ein Ende des derzeitigen Reformprozesses hätte, wie die alternativen Szenarien aufzeigen, dagegen langfristig restriktive Folgen für den Lebensstandard und die finanzielle Basis unserer Sozialsysteme. Der Unterschied zwischen dem Reformszenario „Dänemark“ und 
dem pessimistischeren Szenario einer nur unvollständig realisierten Agenda 2010 („Slow-Go“) beträgt 2,1 Mio. Erwerbstätige im Jahr 2030. Deren Arbeitskraft ist unverzichtbar für unseren Lebensstandard und unsere Sozialversicherungen.

\section{Executive summary}

Demographic change will lead to an increasing number of older people while the number of young people will decline. This change will have deep socio-political consequences regarding the sustainability of our social security systems, will affect the economy as a whole, and might therefore precipitate deep structural changes during the coming decades. The extent of these changes depends not only on the demographic ratio of older to younger people but also on the ratio of economically inactive to economically active individuals.

A projection of this ratio requires medium- and long-term labor force projections. This paper provides such projections for Germany.

The foundation for projections of the future size of the labor force is the demographic development. In light of the past rapid and unbroken increase of life expectancy and the most recent results of international demographic research, we find that official forecasts of future life expectancy are far too pessimistic and predict too short life spans. We therefore first present different demographic scenarios and select the most realistic one as the reference scenario for our labor force projections.

Apart from the underlying demographics, the future size of the labor force is determined by the development of age and gender-specific participation rates. These depend on future political decisions and corresponding behavioral changes. They are thus hard to predict. Hence, we pursue a scenario-based approach in this respect, too.

We select four scenarios to reflect the range of future possible developments. The upper boundary is determined by the size of the working-age population that is given by the underlying demographic projections. In our optimistic scenario we assume that this labor force potential is tapped completely. The lower boundary is marked by the status quo, i.e. the assumption that current participation rates will not change in the future. A reverse trend to even lower participation rates seems implausible to us.

In order to define a realistic scenario within these lower and upper limits, we use the example of one of Germany's neighbors. Denmark is a country which has achieved relatively high labor force participation rates of both women and older people without changing the social contract. Our "Denmark" scenario therefore assumes convergence of the German to the Danish participation rates. In this case, a large part of the demographic pressure can be cushioned through the labor market. This is an important message for economic policy.

Of course one can think of numerous other scenarios, especially decreasing or reversing efforts in the current reform activities short of the complete standstill depicted in the "Status quo" scenario. We model this with a scenario which we call "Slow-Go". This scenario assumes that the reform package of the Agenda 2010 will only be implemented partially. The reader can create additional scenarios by approximate interpolation of our results.

Our main findings are that the size of the labor force and actual employment will decline in the future even in the most favorable scenario - this happens after 2020 at the latest due to the reduction in population size. Neither the optimal utilization of the potential labor supply nor increased net migration may stop this trend completely. In the Denmark scenario the size of the labor force will shrink by around 2.8 million to approximately 39.4 million in 2040 . Without any further labor market reforms, the labor force will contract by more than 9 to around 32.6 million in 2040 . Regarding actual employment, the decline is more moderate in the Denmark scenario (by 2.5 to 36.2 million in 2040) but much stronger in the Status quo scenario (by 11 to 27.4 million in 2040). Hence, an important insight from our work is that targeted reforms which create the necessary incentives for an increase in labor force participation will significantly reduce the adverse forces of demographic change.

At the same time, the German labor force will age rapidly. We find that the share of workers aged 55 and older will increase from around 11 today to roughly $20 \%$ in 2040.

Within a realistic scenario range, our results can be shown to be very robust. Whichever future development will be realized in the end, it can not be a surprise since the natural upper and lower boundaries are well known ("Status quo" and "Best Case"). It can also show that besides shorter education times and the resulting lower average entry age, higher female participation rates and an increase in the retirement age have large impacts on the labor supply.

In summary, this paper shows that a better use of the labor force potential can cushion the consequences of population aging considerably. In particular, the pressures on the social security systems can be mitigated by a modest and gradual reform policy as we model in our Denmark scenario. In turn, stopping the current reform process would have restrictive long-run consequences for the standard of living and the financial basis of the social security systems in Germany. The difference in actual employment between the Denmark scenario and the more pessimistic "Slow-Go" scenario amounts to 2.1 million workers in the year 2030. This manpower will be indispensible in order to maintain the standard of living and the financial basis of the German social security systems. 
Danksagungen Wir danken Anette Reil-Held, Matthias Weiss und zwei anonymen Gutachtern für wertvolle Kommentare, sowie Reinhold Zahn vom Statistischen Bundesamt für detaillierte Auskünfte und zusätzliches Datenmaterial zur 11. koordinierten Bevölkerungsvorausberechnung. Unser Dank gilt ferner den Förderern des MEA für die Unterstützung bei der Erstellung dieses Beitrags, vor allem dem Land Baden-Württemberg, der Deutschen Forschungsgemeinschaft und dem Gesamtverband der Deutschen Versicherungswirtschaft.

\section{Literatur}

Agenda 2010: http://archiv.bundesregierung.de/artikel/81/557981/ attachment/557980_0.pdf (2003). Letzter Zugriff: Oktober 2007

Allmendinger, J., Ebner, C.: Demographischer Wandel. Chancengleichheit für Frauen auf dem Arbeitsmarkt - was ist zu tun? In: Demographischer Wandel - Herausforderung und Chance für den Arbeitsmarkt. Dokumentation ESF Kongress Hessen 2005, 2. September 2005, Wiesbaden (2005)

Berkel, B., Börsch-Supan, A.: Renteneintrittsentscheidungen in Deutschland: Langfristige Auswirkungen verschiedener Reformoptionen. MEA Arbeitspapier 31-03, MEA, Universität Mannheim, Mannheim (2003)

Börsch-Supan, A.: Incentive effects of social security on labour force participation: Evidence in Germany and across Europe. J. Public Econ. 78, 25-49 (2000)

Börsch-Supan, A.: Mehr Zuwanderung? Zur Rolle des Auslands bei der Stabilisierung der gesetzlichen Rentenversicherung in Deutschland, MEA Arbeitspapier 22-02, MEA, Universität Mannheim, Mannheim (2002)

Börsch-Supan, A.: Labour Market Effects of Population Aging. Rev. Labour Econ. Ind. Relat. 17(Special Issue), 5-44 (2003)

Börsch-Supan, A., Wilke, C.: The German Social Security System: How it Was and How it Will Be. MRRC Discussion Paper UM0301, Michigan Retirement Research Center, University of Michigan, Ann Arbor (2003)

Bothfeld, S., Klammer, U., Klenner, C.: WSI-FrauenDatenReport 2005, Handbuch zur wirtschaftlichen und sozialen Situation von Frauen. Edition Sigma, Berlin (2005)

Brussig, M., Wojtkowski, S.: Durchschnittliches Renteneintrittsalter steigt weiter. Altersübergangsreport 2006-02, Hans-BöcklerStiftung, Düsseldorf, und Institut Arbeit und Technik, Gelsenkirchen (2006)

Bucher-Koenen, T., Wilke, C.: Zur Anhebung der Altersgrenzen: Eine Simulation der langfristigen Auswirkungen auf die gesetzliche Rentenversicherung bei unterschiedlichem Renteneintrittsverhalten. MEA Arbeitspapier 159-08, MEA, Universität Mannheim, Mannheim (2008)

Buchheim, C.: Industrielle Revolutionen. dtv, München (1994)

Carone, G.: Long-term labour force projections fort he $25 \mathrm{EU}$ Member States: A set of data for assessing the economics impact of ageing. European Commission Economic Papers, Nr. 235 (2005)

Clemens, J.: Anmerkungen zur geplanten Anhebung des gesetzlichen Rentenalters. Wirtschaftsdienst 3, 163-167 (2006)

Dressel, C.: Erwerbstätigkeit - Arbeitsmarktintegration von Frauen und Männern. In: Bundesministerium für Familie, Senioren, Frauen und Jugend (Hrsg.) 1. Datenreport zur Gleichstellung von Frauen und Männern in der Bundesrepublik Deutschland. Berlin (2005)

Dressel, C., Cornelißen, W., Wolf, K.: Vereinbarkeit von Familie und Beruf. In: Bundesministerium für Familie, Senioren, Frauen und Jugend (Hrsg.) 1. Datenreport zur Gleichstellung von Frauen und Männern in der Bundesrepublik Deutschland. Publikationsverband der Bundesregierung, Rostock (2005)
European Commission: Employment in Europe 2000. Luxemburg (2000)

Friedman, M.: The role of monetary policy. Am. Econ. Rev. 58, 1-17 (1968)

Fuchs, J., Dörfler, K.: Projektion des Erwerbspersonenpotenzials bis 2050 - Annahmen und Datengrundlage. IAB Forschungsbericht Nr. 25/2005. Nürnberg (2005a)

Fuchs, J., Dörfler, K.: Projektion des Arbeitsangebots bis 2050 - Demografische Effekte sind nicht mehr zu bremsen. IAB Kurzbericht Nr. 11/2005. Nürnberg (2005b)

Fuchs, J., Söhnlein, D.: Einflussfaktoren auf das Erwerbspersonenpotenzial - Demografie und Erwerbsverhalten in Ost- und Westdeutschland. IAB Discussion Paper Nr. 12/2007. Nürnberg (2007)

Kommission für die Nachhaltigkeit in der Finanzierung der Sozialen Sicherungssysteme: Nachhaltigkeit in der Finanzierung der Sozialen Sicherungssysteme, Bericht der Kommission. Bundesministerium für Gesundheit und Soziale Sicherung, Berlin (2003)

Reinberg, A., Hummel, M.: Bildungspolitik: Steuert Deutschland langfristig auf einen Fachkräftemangel hin? IAB Kurzbericht Nr. 9/2003. Nürnberg (2003)

Riphahn, R.T., Schmidt, P.: Determinanten des Ruhestandes: Lockt der Ruhestand oder drängt der Arbeitsmarkt? Jahrb. Wirtschaftswiss. 48(1), 113-147 (1997)

Schmidt, P.: Die Wahl des Rentenalters - Theoretische und empirische Analyse des Rentenzugangsverhaltens in West- und Ostdeutschland. Lang, Frankfurt (1995)

Schnabel, S., Kistowski, K., Vaupel, J.: Immer neue Rekorde und kein Ende in Sicht. In: Hoern, J.M., Vaupel, J.W. (Hrsg.) Demografische Forschung - Aus Erster Hand. 2. Jg., Bd. 2. Max-PlanckInstitut für demographische Forschung, Rostock (2005)

Schnur, P., Zika, G.: Projektion des Arbeitskräftebedarfs bis 2020 - Nur zögerliche Besserung am deutschen Arbeitsmarkt. IAB Kurzbericht Nr. 12/2005. Nürnberg (2005)

Siddiqui, S.: The Pension Incentive to Retire: Empirical Evidence for West Germany. J. Popul. Econ. 10(4), 463-86 (1997)

Statistisches Bundesamt: 11. koordinierte Bevölkerungsvorausberechnung - Annahmen und Ergebnisse. Statistisches Bundesamt, Wiesbaden, www.destatis.de (2006). Letzter Zugriff: Oktober 2007

Statistics Denmark: www.statbank.dk. Letzter Zugriff: Oktober 2007

Wissenschaftlicher Beirat beim Bundesministerium für Wirtschaft und Arbeit: Alterung und Familienpolitik. Bundesministerium für Wirtschaft und Arbeit, Berlin (2005)

Axel Börsch-Supan, Studium der Volkswirtschaftslehre und Mathematik in München und Bonn. 1980 Abschluss als Diplom-Mathematiker. 1984 Promotion am M.I.T. in Cambridge (USA). 1984-1989 Professur für Public Policy in Harvard. 1989-2001 Professur für Makroökonomik und Wirtschaftspolitik der Universität Mannheim. Seit 2001 Direktor des von ihm gegründeten Mannheimer Forschungsinstituts Ökonomie und Demografischer Wandel (MEA). Vorsitzender des wissenschaftlichen Beirats beim Bundesministerium für Wirtschaft und Arbeit. Mitglied der Berlin-Brandenburgischen Akademie der Wissenschaften und der Deutschen Nationalakademie Leopoldina.

Forschungsfelder: Auswirkungen des demografischen Wandels, der Reform der sozialen Sicherungssysteme sowie Arbeits- und Kapitalmarktfragen.

E-Mail: Axel@Boersch-Supan.de

Christina Benita Wilke, Studium der Betriebs- und Volkswirtschaftslehre an den Universitäten Passau, Lund (Schweden), Mannheim und 
Hagen. 2002 Abschluss als Diplom-Kauffrau. 2007 Abschluss als Diplom-Volkswirtin. Seit November 2002 wissenschaftliche Mitarbeiterin im Bereich „Alterssicherung und Sparen“ am Mannheimer Forschungsinstitut Ökonomie und Demografischer Wandel (MEA) und seit September 2008 Geschäftsführerin des Instituts.
Forschungsfelder: Auswirkungen des demografischen Wandels, Prognosen zur künftigen Bevölkerungs- und Arbeitsmarktentwicklung, Rentensysteme und -reformen, Alterseinkommen und private Altersvorsorge, Simulationsmodelle.

E-Mail: wilke@mea.uni-mannheim.de 